TRANSACTIONS OF THE

AMERICAN MATHEMATICAL SOCIETY

Volume 354, Number 4, Pages 1393-1420

S 0002-9947(01)02882-3

Article electronically published on November 8, 2001

\title{
SECOND ORDER LAGRANGIAN TWIST SYSTEMS: SIMPLE CLOSED CHARACTERISTICS
}

\author{
J. B. VAN DEN BERG AND R. C. VANDERVORST
}

\begin{abstract}
We consider a special class of Lagrangians that play a fundamental role in the theory of second order Lagrangian systems: Twist systems. This subclass of Lagrangian systems is defined via a convenient monotonicity property that such systems share. This monotonicity property (Twist property) allows a finite dimensional reduction of the variational principle for finding closed characteristics in fixed energy levels. This reduction has some similarities with the method of broken geodesics for the geodesic variational problem on Riemannian manifolds. On the other hand, the monotonicity property can be related to the existence of local Twist maps in the associated Hamiltonian flow.

The finite dimensional reduction gives rise to a second order monotone recurrence relation. We study these recurrence relations to find simple closed characteristics for the Lagrangian system. More complicated closed characteristics will be dealt with in future work. Furthermore, we give conditions on the Lagrangian that guarantee the Twist property.
\end{abstract}

\section{INTRODUCTION}

Various mathematical models for problems in nonlinear elasticity, nonlinear optics, solid mechanics, etc. are derived from second order Lagrangian principles, i.e., the differential equations are obtained as the Euler-Lagrange equations of a Lagrangian $L$ that depends on a state variable $u$, and its first and second order derivatives. The Euler-Lagrange differential equations are fourth order and are of conservative nature. In scalar models the Lagrangian action is defined by $J[u]=\int L\left(u, u^{\prime}, u^{\prime \prime}\right) d t$. A second order Lagrangian system is, under suitable assumptions on the $u^{\prime \prime}$-dependence of $L$, equivalent to a Hamiltonian system on $\mathbb{R}^{4}$. Trajectories of the Lagrangian system, and thus Hamiltonian system, lie on three dimensional sets $M_{E} \stackrel{\text { def }}{=}\{H=E\}$, where $H$ is the Hamiltonian (conserved quantity). The sets $M_{E}$ are smooth manifolds for all regular $E$ values of $H$ (i.e. $\left.\nabla H\right|_{M_{E}} \neq 0$ ), and are non-compact for all $E \in \mathbb{R}$. It turns out that for Hamiltonian systems that come from second order Lagrangians, one can find a natural two dimensional section $\left\{u^{\prime}=0\right\} \cap M_{E}$ which bounded trajectories have to intersect finitely or infinitely many times (possibly only in the limit) [19]. This section will be denoted

Received by the editors January 18, 2000.

1991 Mathematics Subject Classification. Primary 34C12, 49Jxx, 49S05, 70Hxx, 70Kxx.

The first author was supported by grants TMR ERBFMRXCT980201 and NWO SIR13-4785.

The second author by grants ARO DAAH-0493G0199 and NIST G-06-605. 
by $\Sigma_{E}$ and $\Sigma_{E}=N_{E} \times \mathbb{R}$, where $N_{E}$ is a one dimensional set defined by

$$
N_{E}=\left\{\left(u, u^{\prime \prime}\right) \mid \frac{\partial L}{\partial u^{\prime \prime}} u^{\prime \prime}-L\left(u, 0, u^{\prime \prime}\right)=E\right\}
$$

(see Section 1.1 for more details).

The Hamiltonian flow induces a return map to the section $\Sigma_{E}$, and closed trajectories - closed characteristics - correspond to fixed points of iterates of this map. In many situations the return map is an analogue of a monotone area-preserving Twist map (see e.g. 6, 22, 25]). The theory developed in this paper will be centered around this property. Lagrangian systems that allow such Twist maps will be referred to as Twist systems. Definitions and a more precise analysis will be given in the forthcoming sections. This paper will be concerned with the basic properties of Twist systems and the study of simple closed characteristics. These are periodic trajectories that, when represented in the $\left(u, u^{\prime}\right)$-plane (configuration plane of the Lagrangian system), are simple closed curves. In [12, 13] we will investigate more elaborate types of closed characteristics via a Morse type theory in relation with braiding and knotting of trajectories. One of the main results of this paper is the following.

Theorem 1. Consider a Twist system (Definition 3) with Lagrangian L $\left(u, u^{\prime}, u^{\prime \prime}\right)$, and let $E$ be a regular value. If $N_{E}$ has a compact connected component $N_{E}^{c}$, then there exists at least one simple closed characteristic at energy level $E$ with $u(t) \in \pi^{u} N_{E}$ for all $t \in \mathbb{R}$ (where $\pi^{u} N_{E}$ is the projection of $N_{E}$ onto the $u$ coordinate).

A precise statement of this result will be presented in Section 3.1 together with information about the location and the Morse index of the trajectory (Theorem 12). The results in this paper are proved for Twist systems. We can safely conjecture that Theorem 1 remains true even without the Twist property. This can for example be achieved via continuation to a Twist system within the class of simple closed curves in the $\left(u, u^{\prime}\right)$-plane. This exploits the idea that no simple closed characteristics exist on the boundary of the class of simple closed curves. Certain mild growth conditions on $L$ are needed in this case (also for the continuation). This idea will be the subject of future study, see [16].

For singular energy levels a similar theorem can be proved (Theorem 14). The bottom line is that under the same compactness assumptions there exists a simple closed characteristic in the broader sense of the word, i.e., depending on possible singularities a closed characteristic is either a regular simple closed trajectory, a simple homoclinic loop, or a simple heteroclinic loop. We also explain how singularities can lead to multiplicity of closed characteristics (this issue is addressed in full in 12] and [13]). In Section 4 we give some more background information on Twist maps including a few observations deduced from numerical calculations. We also briefly discuss the analogues of KAM-tori/circles for second order Lagrangian systems, and the issue of integrability versus non-integrability. Throughout the paper specific examples of physical systems will be given such as the extended Fisher-Kolmogorov (eFK) and Swift-Hohenberg equations $\left(u^{\prime \prime \prime \prime}-\alpha u^{\prime \prime}+F^{\prime}(u)=0\right.$ with $\alpha \in \mathbb{R}$ ). The theory developed here also applies to systems on $M=S^{1}$ by simply assuming $L$ to be periodic in $u$.

The organization of the paper is as follows. We introduce the concepts that play a major role in our analysis in Sections 1.1 1.3 The definition of the Twist 
property is stated in Section [2.1. Some examples of Twist systems are given in Section 2.2 and in Section 2.3 we explain to what extent the assumptions can be weakened. Subsequently, we apply the theory to Twist systems on energy levels with (Sections [3.2] and [3.3) and without singular points (Sections [3.1). We deal with non-compact interval components in Section 3.4. In Section 4 we list some concluding remarks. Finally, the appendices are devoted to the classification of equilibrium points and the proof of the Twist property for a specific class of second order Lagrangians.

1.1. Second order Lagrangians. Let $L: \mathbb{R}^{3} \rightarrow \mathbb{R}$ be a $C^{2}$-function of the variables $u, v, w$. For any smooth function $u: I \rightarrow \mathbb{R}, I \subseteq \mathbb{R}$, define the functional $J[u]=\int_{I} L\left(u, u^{\prime}, u^{\prime \prime}\right) d t$, which is called the (Lagrangian) action of $u$. The function $L$ may be regarded as a function on 2 -jets of functions $u: \mathbb{R} \rightarrow \mathbb{R}$, and is generally referred to as the Lagrangian 1 . The pair $(L, d t)$ is called a second order Lagrangian system on $\mathbb{R}$. The action $J$ of the Lagrangian system is said to be stationary at a function $u$ if $\delta J[u]=0$ with respect to variations $\delta u \in C_{c}^{\infty}(I, \mathbb{R}) \sqrt{2}$, i.e.

$$
\begin{aligned}
\delta J[u] & =\delta \int_{I} L\left(u, u^{\prime}, u^{\prime \prime}\right) d t=\int_{I}\left[\frac{\partial L}{\partial u} \delta u+\frac{\partial L}{\partial v} \delta u^{\prime}+\frac{\partial L}{\partial w} \delta u^{\prime \prime}\right] d t \\
& =\int_{I}\left[\frac{\partial L}{\partial u}-\frac{d}{d t} \frac{\partial L}{\partial v}+\frac{d^{2}}{d t^{2}} \frac{\partial L}{\partial w}\right] \delta u d t=0 .
\end{aligned}
$$

A stationary function $u$ thus satisfies the differential equation

$$
\frac{\partial L}{\partial u}-\frac{d}{d t} \frac{\partial L}{\partial u^{\prime}}+\frac{d^{2}}{d t^{2}} \frac{\partial L}{\partial u^{\prime \prime}}=0,
$$

which is called the Euler-Lagrange equation of the Lagrangian system $(L, d t)$. The Lagrangian action $J$ is invariant under the $\mathbb{R}$-action $t \mapsto t+c$, which by Noether's Theorem yields the conservation law

$$
\left(\frac{\partial L}{\partial u^{\prime}}-\frac{d}{d t} \frac{\partial L}{\partial u^{\prime \prime}}\right) u^{\prime}+\frac{\partial L}{\partial u^{\prime \prime}} u^{\prime \prime}-L\left(u, u^{\prime}, u^{\prime \prime}\right)=\text { constant }
$$

(see for instance [21]). This conservation law is called the Hamiltonian.

If $L$ is strictly convex in the $w$-variable then the Lagrangian system $(L, d t)$ is equivalent to a Hamiltonian system on $\mathbb{R}^{4}$ with the standard symplectic structure. Therefore we assume:

(H) $\partial_{w}^{2} L(u, v, w) \geq \delta>0$ for all $(u, v, w)$.

The correspondence between a Lagrangian system $(L, d t)$ on $\mathbb{R}$ and a Hamiltonian system $(H, \omega)$ on $\mathbb{R}^{4}$ can be explained as follows. Let $x=\left(p_{u}, p_{v}, u, v\right)$ be symplectic coordinates for $\mathbb{R}^{4}$ with the symplectic form given by $\omega=d p_{u} \wedge d u+d p_{v} \wedge d v$. Define the Hamiltonian $H(x)=p_{u} v+L^{*}\left(u, v, p_{v}\right)$, where

$$
L^{*}\left(u, v, p_{v}\right)=\max _{w \in \mathbb{R}}\left\{p_{v} w-L(u, v, w)\right\}
$$

is is the Legendre transform of $L$. Since $L$ is strictly convex in $w$ we have that $L^{*}$ is strictly convex in $p_{v}$. Moreover, $\partial_{p_{v}} L^{*}=\left(\partial_{w} L\right)^{-1}\left(p_{v}\right)=w$, hence $H(x)=$

\footnotetext{
${ }^{1}$ In the case of a general smooth 1-dimensional manifold $M$ one defines $L$ as a smooth function on 2 -jets of functions $u: \mathbb{R} \rightarrow M$. The action is then defined by considering functions $\left(u, u^{\prime}, u^{\prime \prime}\right): I \rightarrow J^{2} M$.
}

${ }^{2}$ If $M$ is an arbitrary 1-dimensional manifold a different notion of variation is used. 
$p_{u} v+p_{v}\left(\partial_{w} L\right)^{-1}\left(p_{v}\right)-L\left(u, v,\left(\partial_{w} L\right)^{-1}\left(p_{v}\right)\right)$. For any function $x: I \rightarrow \mathbb{R}$ the Hamiltonian action is defined by $\mathcal{A}[x]=\int_{I}\left[p_{u} u^{\prime}+p_{v} v^{\prime}-H(x)\right] d t$. A function $x$ is stationary for $\mathcal{A}$ if and only if the $u$-coordinate is stationary for $J$. In particular, the Euler-Lagrange equations for $\mathcal{A}$ are of the form $x^{\prime}=X_{H}(x)$, where $X_{H}=\mathcal{J} \nabla H$ and $\mathcal{J}$ is defined by $\omega(x, \mathcal{\partial} y)=\langle x, y\rangle$ (where $\langle x, y\rangle$ is the standard inner product in $\mathbb{R}^{4}$ ). $X_{H}$ is called the Hamiltonian vector field associated to $H$. The correspondence between $u$ and its derivatives and $x$ is given by: $v=u^{\prime}, p_{u}=\partial_{u^{\prime}} L-p_{v}^{\prime}$, and $p_{v}=\partial_{u^{\prime \prime}} L$. See for example [4] for more details on this correspondence. The state space $\mathbb{R}^{4}$ of the Hamiltonian system $(H, \omega)$ is often referred to as the phase space and $J^{1} \mathbb{R} \simeq \mathbb{R}^{2}$ is called the configuration space 3 .

If the Hamiltonian is sufficiently smooth, then the Hamiltonian system $x^{\prime}=$ $X_{H}(x)$ generates a local flow on $\mathbb{R}^{4}$. If we assume strict convexity of $L$ in the $w$-variable then $H$ is of class $C^{1}$. Under Hypothesis $(\mathrm{H})$ the Hamiltonian $H(x)$ is a $C^{2}$-function, which in turn generates a local $C^{1}$-flow $\phi_{H}^{t}$ on $\mathbb{R}^{4}$ via the equation $x^{\prime}=X_{H}(x)$.

Stationary functions of $J$ satisfy Equation (2), which is equivalent to $H(x)=$ $E \in \mathbb{R}$. For the associated Hamiltonian system $(H, \omega)$ this means that the stationary motions lie on the 3 -dimensional sets $M_{E}=\left\{x \in \mathbb{R}^{4} \mid H(x)=E\right\}$. If $\nabla H \neq 0$ on $M_{E}$, then $E$ is called a regular value and $M_{E}$ is a smooth non-compact manifold without boundary. The vector field $X_{H}$ restricted to $M_{E}$ is non-singular when $E$ is a regular value. Indeed, the singular points of the vector field $X_{H}$, i.e. points $x_{*}$ such that $X_{H}\left(x_{*}\right)=0$, are exactly the critical points of the Hamiltonian, and thus only occur at singular energy levels. Singular points are of the form $x_{*}=\left(p_{u}, p_{v}, u, 0\right)$ and are given by: $\partial_{u} L(u, 0,0)=0, p_{u}=\partial_{v} L(u, 0,0)$ and $p_{v}=\partial_{w} L(u, 0,0)$. Equivalently, for a Lagrangian system an energy level $E$ is said to be regular if and only if $\frac{\partial L}{\partial u}(u, 0,0) \neq 0$ for all points $u \in \mathbb{R}$ that satisfy the relation $-L(u, 0,0)=E$.

A bounded characteristic of a Lagrangian system $(L, d t)$ is a function $u \in$ $C_{b}^{2}(\mathbb{R}, \mathbb{R})$ for which $\delta \int_{I} L\left(u, u^{\prime}, u^{\prime \prime}\right) d t=0$ with respect to variations $\delta u \in C_{c}^{2}(I, \mathbb{R})$ for any compact interval $I \subset \mathbb{R}$. Since the Lagrangian is a $C^{2}$-function of the variables $(u, v, w)$ it follows from the Euler-Lagrange equations that $u \in C_{b}^{3}(\mathbb{R}, \mathbb{R})$, $\frac{\partial L}{\partial w}(\cdot) \in C_{b}^{2}(\mathbb{R}, \mathbb{R})$, and $\left(\frac{d}{d t} \frac{\partial L}{\partial w}-\frac{\partial L}{\partial v}\right)(\cdot) \in C_{b}^{2}(\mathbb{R}, \mathbb{R})$ (regularity of extremals of $L$ ). This is equivalent to having a function $x \in C_{b}^{2}\left(\mathbb{R}, \mathbb{R}^{4}\right)$ which is stationary for $\mathcal{A}[x]$ : a bounded characteristic for the associated Hamiltonian system $(H, \omega)$.

The question now arises, given an energy value $E$, do there exist bounded and/or closed characteristics (see Sections 1.2 and 1.3 for a definition) on $M_{E}$, and how many, and how are these questions related to geometric and topological properties of $M_{E}$.

1.2. Cross-sections and area-preserving maps. From $(H)$ it follows that bounded solutions of the Euler-Lagrange equations only have isolated extrema (wellposedness of the initial value problem for $x^{\prime}=X_{H}(x)$ ). Consequently, a bounded characteristic has either finitely, or infinitely many isolated local extrema. For the associated Hamiltonian system this means that a bounded trajectory always intersects the section $\Sigma_{E}=\{v=0\} \cap M_{E}=\left\{\left(p_{u}, p_{v}, u, 0\right) \mid p_{u} \in \mathbb{R}, p_{v}=\right.$

\footnotetext{
${ }^{3}$ In the general case the configuration space is $J^{1} M$ and the phase space is $T J^{1} M$.

${ }^{4}$ In order to study stationary points of $\mathcal{A}$ additional regularity for $H$ is not required. One does usually need proper growth conditions on $H$.
} 
$\left.\partial_{w} L(u, 0, w),(u, w) \in N_{E}\right\}$, where $N_{E}$ is defined by (1) 5 . In the case that there are only finitely many (or zero) intersections, $x$ must be asymptotic, as $t \rightarrow \pm \infty$, to singular points of $X_{H}$, and thus critical points of $H$. If $E$ is a regular value this possibility is excluded. A bounded solution $u$ is therefore a concatenation of monotone laps between extrema - an increasing lap followed by a decreasing lap and vice versa - at least if we assume that $u$ does not have critical inflection points, i.e. $\Sigma_{E}$ is not intersected in a point where $w=0$. In this context it is important to note that if $E$ is a regular value then critical inflection points can only occur at the boundary of

$$
\pi^{u} N_{E} \stackrel{\text { def }}{=}\left\{u \mid(u, w) \in N_{E} \text { for some } w \in \mathbb{R}\right\}=\{u \mid L(u, 0,0)+E \geq 0\} .
$$

The last equality follows from the definition of $N_{E}$ and the fact that

$$
\partial_{w}\left(w \partial_{w} L-L\right)=w \partial_{w}^{2} L
$$

in combination with hypothesis $(\mathrm{H})$. We will be interested in bounded characteristics that avoid critical inflection points. It will follow later on that at regular energy values critical inflection points cannot occur (Lemma 7 ).

Recalling that $w=\partial_{p_{v}} L^{*}$ we define $N_{E}^{+}=\left\{\left(u, p_{v}\right) \in N_{E} \mid \partial_{p_{v}} L^{*}\left(u, 0, p_{v}\right)>0\right\}$, $N_{E}^{-}=\left\{\left(u, p_{v}\right) \in N_{E} \mid \partial_{p_{v}} L^{*}\left(u, 0, p_{v}\right)<0\right\}, N_{E}^{0}=\left\{\left(u, p_{v}\right) \in N_{E} \mid \partial_{p_{v}} L^{*}\left(u, 0, p_{v}\right)\right.$ $=0\}$. It follows from Hypothesis (H) that $N_{E}^{+}$and $N_{E}^{-}$are smooth graphs over the $u$-axis and $\pi^{u} N_{E}^{+}=\pi^{u} N_{E}^{-}$. The sets $\Sigma_{E}^{ \pm}=N_{E}^{ \pm} \times \mathbb{R}$ are smooth surfaces over the $\left(p_{u}, u\right)$-plane. Thus, the projections $\pi_{ \pm}: \Sigma_{E}^{ \pm} \rightarrow \pi^{u} N_{E}^{ \pm} \times \mathbb{R}$ are invertible. For a given bounded trajectory $x(t)$ we therefore only need to know the $\left(p_{u}, u\right)$ coordinates of the intersections of $x(t)$ with $\Sigma_{E}^{ \pm}$. Consequently bounded characteristics can be identified with sequences of points $\left(p_{u_{i}}, u_{i}\right)$ in the $\left(p_{u}, u\right)$-plane.

In the following we fix the energy level $E$ and drop the subscript in the notation. The vector field $X_{H}$ is tranverse to the section $\Sigma^{+} \cup \Sigma^{-}$(non-transverse at $\Sigma^{0}$ ). It therefore makes sense to consider the Poincaré return maps, i.e. maps from $\Sigma^{+}$to $\Sigma^{-}$and from $\Sigma^{-}$to $\Sigma^{+}$, by following the flow $\varphi_{H}^{t}$ starting at $\Sigma^{+}$until it intersects $\Sigma^{-}$. It may happen that $\varphi_{H}^{t}$ does not intersect $\Sigma^{-}$at all. For the points in $\Sigma^{+}$ for which the flow does intersect $\Sigma^{-}$we define a map $\mathcal{T}_{+}$from $\Sigma^{+}$to $\Sigma^{-6}$. The same can be done for the map $\mathcal{T}_{-}$mapping from $\Sigma^{-}$to $\Sigma^{+}$. Since $\Sigma^{ \pm}$are graphs over the $\left(p_{u}, u\right)$-plane the above defined maps induce maps $T_{ \pm}=\pi_{\mp} \mathcal{T}_{ \pm} \pi_{ \pm}^{-1}$ between open regions $\Omega^{ \pm} \subset \pi_{ \pm} \Sigma^{ \pm}$, i.e. $T_{ \pm}: \Omega^{ \pm} \rightarrow \Omega^{\mp}$ (see also Figure 1). For any point $\left(p_{u}, u\right) \in \Omega^{ \pm}, T_{ \pm}$is a local $C^{1}$-diffeomorphism (since there are no critical inflection points in $N^{ \pm}$).

Since bounded characteristics consist of increasing laps followed by decreasing laps we seek fixed points of iterates of the composition map $T=T_{-} \circ T_{+}$(or $\left.T=T_{+} \circ T_{-}\right)$. Fixed points are contained in the set

$$
\Omega^{*}=\bigcap_{n \in \mathbb{Z}}\left(T_{-} \circ T_{+}\right)^{n}\left(\Omega^{+}\right) \subset \mathbb{R}^{2} .
$$

The maps $T_{ \pm}$are area-preserving maps with respect to the area form $\alpha=d p_{u} \wedge$ $d u$. This means that for any region $U \subset \Omega^{ \pm}$it holds that $\int_{U} \alpha=\int_{T_{ \pm} U} T_{ \pm}^{*} \alpha$ (locally area-preserving). This was proved in 19 for the eFK-equation. We will give a different proof of this fact here. Let $\left(p_{u}, u\right) \in U \subset \Omega^{+}$, and recall that

\footnotetext{
${ }^{5}$ It is sometimes convenient to define $N_{E}$ in terms of coordinates $\left(u, p_{v}\right)$ by simply using the formula $p_{v}=\partial_{w} L$.

${ }^{6}$ In ODE theory the study of this map is often called a shooting method.
} 


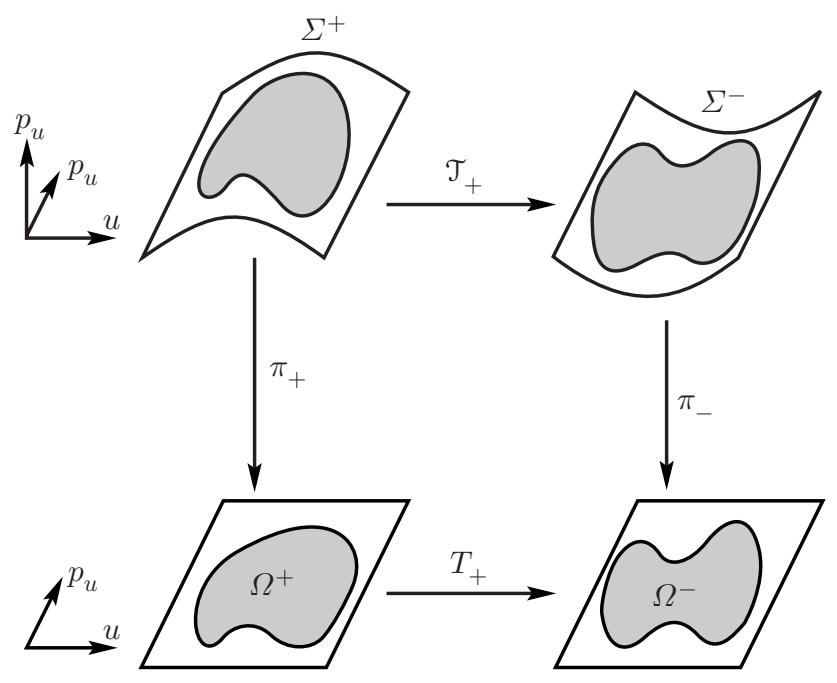

Figure 1. The map $\mathcal{T}_{+}$, which is induced by the flow, and its projection $T_{+}$.

$\omega=d p_{u} \wedge d u+d p_{v} \wedge d v$. Now $\mathcal{T}_{+}$maps $\pi_{+}^{-1} U \subset \Sigma^{+}$to $\mathcal{T}_{+} \pi_{+}^{-1} U \subset \Sigma^{-}$. Since $\mathcal{T}_{+}$ preserves $\omega$, and because $\Sigma^{ \pm} \subset\{v=0\}$ it follows that the 2 -form $\alpha=d p_{u} \wedge d u$ is preserved, and thus $T_{+}$, as a map from $\Omega^{+}$to $\Omega^{-}$, is area-preserving. This implies that

$$
p_{u_{2}} d u_{2}-p_{u_{1}} d u_{1}=d S_{*}\left(p_{u_{1}}, u_{1}\right),
$$

where $\left(p_{u_{1}}, u_{1}\right) \in U$ and $\left(p_{u_{2}}, u_{2}\right)=T_{+}\left(p_{u_{1}}, u_{1}\right) \in T_{+} U$, and $S_{*}$ is a $C^{1}$-function of $\left(p_{u_{1}}, u_{1}\right)$.

The map $T_{+}$is a (local) Twist map if $u_{2}=u_{2}\left(p_{u_{1}}, u_{1}\right)$ is strictly increasing in $-p_{u_{1}}$. It then follows from (3) that there exists a $C^{1}$-function $S_{E}\left(u_{1}, u_{2}\right)=$ $S_{*}\left(p_{u_{1}}\left(u_{1}, u_{2}\right), u_{1}\right)$ such that $\partial_{1} S_{E}=-p_{u_{1}}$ and $\partial_{2} S_{E}=p_{u_{2}}$. This function is called the generating function of the Twist map. A similar construction can be carried out for $T_{-}$. We refer to 5 , for more details.

The function $S_{E}$ can be used to formulate a variational principle in terms of the $u_{i}$-variables. In the next chapter we will make a connection with the variational principle for the Lagrangian action

$$
J_{E}[u]=\int_{0}^{\tau}\left(L\left(u, u^{\prime}, u^{\prime \prime}\right)+E\right) d t
$$

where the integration over $[0, \tau]$ is between two consecutive extrema of $u(t)$. In relation to this connection we note the following (which does not depend on $T_{+}$ being a Twist map or not).

Lemma 2. Let $S_{*}\left(p_{u_{1}}, u_{1}\right)=J_{E}[u]$, where $u(t)$ is the trajectory starting at $\pi_{+}^{-1}\left(p_{u_{1}}, u_{1}\right) \in \Sigma^{+}$, and $\tau=\tau\left(p_{u_{1}}, u_{1}\right)$ is the first intersection time at $\Sigma^{-}$. Then $S_{*}$ satisfies Equation (3).

Proof. Define the Hamiltonian action $\mathcal{A}_{E}[x]=\int_{0}^{T}\left\{p_{u} u^{\prime}+p_{v} v^{\prime}-H(x)+E\right\} d t$, and let $\left(p_{u_{1}}, u_{1}\right) \in \Omega^{+}$. Consider the trajectory $\left\{\varphi_{H}^{t}\left(\pi_{+}^{-1}\left(p_{u_{1}}, u_{1}\right)\right)\right\}_{t=0}^{t=\tau\left(p_{u_{1}}, u_{1}\right)}$, where $\tau\left(p_{u_{1}}, u_{1}\right)$ is the first intersection time at $\Sigma^{-}$. These trajectories vary smoothly with 
$\left(p_{u_{1}}, u_{1}\right) \in \Omega^{+}$. We now consider variations with respect to $\left(p_{u_{1}}, u_{1}\right) \in \Omega^{+}$. Using the fact that $\left(p_{u}, p_{v}, u, v\right)$ obeys the Hamilton equations and $v\left(\tau\left(p_{u_{1}}, u_{1}\right)\right)=0$, we obtain

$$
\begin{aligned}
\delta \mathcal{A}_{E}[x] & =\left.p_{u} \delta u\right|_{0} ^{\tau}+\left.p_{v} \delta v\right|_{0} ^{\tau}+\left[p_{u} u^{\prime}+p_{v} v^{\prime}-H(x)+E\right]_{\tau} \delta \tau \\
& =p_{u} \delta u(\tau)-p_{u} \delta u(0)+p_{v}\left[\delta v(\tau)+v^{\prime}(\tau) \delta \tau\right] \\
& =p_{u_{2}} \delta u_{2}-p_{u_{1}} \delta u_{1},
\end{aligned}
$$

where $\left(p_{u_{2}}, u_{2}\right)=T_{+}\left(p_{u_{1}}, u_{1}\right)$. It may be clear that $\mathcal{A}_{E}[x]=J_{E}[u]$, which proves the lemma.

If $T_{+}$is a Twist map then for $J_{E}$ this implies that there exists a local continuous family $u\left(t ; u_{1}, u_{2}\right)$ of extremals (and $\tau\left(u_{1}, u_{2}\right)$ varies continuously). Conversely, we will show in the next chapter that the continuity conditions on the family of extremals $u\left(t ; u_{1}, u_{2}\right)$ imply the Twist property.

We remark that instead of studying the maps $T_{ \pm}$one can study a related areapreserving map which is well defined when $T_{ \pm}$are Twist maps. From $T_{ \pm}$we construct the map $\tilde{T}$

$$
\left(\begin{array}{c}
u_{n+1} \\
u_{n}
\end{array}\right)=\tilde{T}\left(\begin{array}{c}
u_{n} \\
u_{n-1}
\end{array}\right), \quad u_{n-1}, u_{n}, u_{n+1} \in \pi^{u} N_{E}
$$

For this map we can use the generating function $S_{E}\left(u_{1}, u_{2}\right)$ to retrieve the maps $T_{ \pm}$. We refer to 3 6] for more details.

1.3. Closed characteristics. A special class of bounded characteristics are closed characteristics. These are functions $u$ that are stationary for $J[u]$ and are $\tau$-periodic for some period $\tau$. If we seek closed characteristics at a given energy level $E$ we can invoke the following variational principle:

$$
\text { Extremize }\left\{J_{E}[u] \mid u \in \Omega_{\text {per }}\right\},
$$

where $J_{E}[u]=\int_{0}^{\tau}\left(L\left(u, u^{\prime}, u^{\prime \prime}\right)+E\right) d t$ and $\Omega_{\text {per }}=\bigcup_{\tau>0} C^{2}\left(S^{1}, \tau\right)$. It may be clear that $\tau$ is also a parameter in this problem. Problem (4) is equivalent to

$$
\text { Extremize }\left\{J_{E}[v, \tau] \mid(v, \tau) \in C^{2}\left(S^{1}, 1\right) \times \mathbb{R}^{+}\right\},
$$

where $J_{E}[v, \tau]=\int_{0}^{1}\left(L\left(v, \frac{v^{\prime}}{\tau}, \frac{v^{\prime \prime}}{\tau^{2}}\right)+E\right) \tau d s$. This equivalent variational characterization is convenient for technical purposes. Notice that the variations in $\tau$ guarantee that any extremal of (4) has energy $H(x)=E$. The variational problem of finding closed characteristics for a given energy value $E$ can also be formulated in terms of unparametrized closed curves in the configuration plane.

The Morse index of a closed characteristic $u$ is defined as the number of negative eigenvalues of the linear operator $d^{2} J_{E}[u]$ on $T_{u} \Omega_{\text {per }} \simeq C^{2}\left(S^{1}, 1\right) \times \mathbb{R}$. The nullity is the dimension of the kernel of $d^{2} J_{E}[u]$. The large Morse index is defined as the sum of the Morse index and the nullity.

\section{TWIST SYSTEMS}

2.1. Generating functions. In this section we will introduce a class of Lagrangian systems which satisfy a variant of the Twist property. Such systems can be studied via generating functions. We start with systems for which the generating function is of class $C^{2}$. In Section 2.2 we will give a number of examples of such systems. In Section 2.3 we explain how the theory also works with $C^{1}$-generating functions 
which allows a weaker version of the Twist property (see Hypothesis $\left(\mathrm{T}^{\prime}\right)$ in Section [2.3).

For a regular energy value $E$ the set $\pi^{u} N_{E}$ is a union of closed intervals. Connected components of $\pi^{u} N_{E}$ are denoted by $I_{E}$ and will be referred to as interval components. Since $E$ is regular it holds that $L(u, 0,0)+E>0$ for $u \in \operatorname{int}\left(I_{E}\right)$, and $L(u, 0,0)+E=0$ for $u \in \partial I_{E}$. In terms of $N_{E}$ this means that connected components of $N_{E}$ topologically are copies of $\mathbb{R}$ and/or $S^{1}$. Let $\Delta=\left\{\left(u_{1}, u_{2}\right) \in\right.$ $\left.I_{E} \times I_{E} \mid u_{1}=u_{2}\right\}$ be the diagonal, then for any pair $\left(u_{1}, u_{2}\right) \in I_{E} \times I_{E} \backslash \Delta$ we define

$$
S_{E}\left(u_{1}, u_{2}\right)=\inf _{\substack{u \in X_{\tau} \\ \tau \in \mathbb{R}^{+}}} \int_{0}^{\tau}\left(L\left(u, u^{\prime}, u^{\prime \prime}\right)+E\right) d t,
$$

where $X_{\tau}=X_{\tau}\left(u_{1}, u_{2}\right)=\left\{u \in C^{2}([0, \tau]) \mid u(0)=u_{1}, u(\tau)=u_{2}, u^{\prime}(0)=u^{\prime}(\tau)=\right.$ $0,\left.u^{\prime}\right|_{(0, \tau)}>0$ if $u_{1}<u_{2}$ and $\left.u^{\prime}\right|_{(0, \tau)}<0$ if $\left.u_{1}>u_{2}\right\}$. We remark that the notation $S_{E}$ is slightly suggestive since it is not a priori clear that this definition of $S_{E}$ is equivalent to the one in Section 1.2 (however, compare Lemma 2). If there is no ambiguity about the choice of $E$ we simply write $S\left(u_{1}, u_{2}\right)$. At this point it is not clear whether $S$ is defined on all of $I_{E} \times I_{E} \backslash \Delta$.

Collections of monotone pieces, or laps, of $u$ from $u_{1}$ to $u_{2}$ that minimize $\int(L+E)$ are the analogues of broken geodesics. Our goal now is to formulate a variational problem in terms of the $u_{i}$-coordinates of bounded characteristics replacing the 'full' variational problem for $J_{E}[u]$. This will be a direct analogue of the method of broken geodesics.

As in (5) there is an equivalent formulation of the variational problem above. In view of this we consider the pair $(v, \tau)$, with $v(s)=u(t)$ and $s=t / \tau$. For the special points $\left(u_{1}, u_{2}\right) \in \Delta$ we define $v(s)=u_{1}$ for all $s \in[0,1]$ and $\tau=0$ (and $\left.S\left(u_{1}, u_{1}\right)=0\right)$. A Lagrangian system $(L, d t)$ is said to satisfy the Twist property on an interval component $I_{E}$ if (with $E$ a regular energy value):

$(\mathrm{T}) \inf \left\{J_{E}[u] \mid u \in X_{\tau}\left(u_{1}, u_{2}\right), \tau \in \mathbb{R}^{+}\right\}$has a minimizer $u\left(t ; u_{1}, u_{2}\right)$ for all $\left(u_{1}, u_{2}\right) \in I_{E} \times I_{E} \backslash \Delta$, and $u$ and $\tau$ are $C^{1}$-smooth functions of $\left(u_{1}, u_{2}\right)$.

To be precise, by $C^{1}$-smoothness we mean that $\left(u_{1}, u_{2}\right) \rightarrow(v, \tau)$ is a $C^{1}$-function from $\operatorname{int}\left(I_{E} \times I_{E} \backslash \Delta\right)$ to $C^{2}([0,1]) \times \mathbb{R}^{+}$and a $C^{0}$-function on $I_{E} \times I_{E}$. The results presented in this paper will apply whenever the Twist property is satisfied on an interval component $I_{E} 7$.

If $E$ is a singular energy level with non-degenerate critical points, then we have the same formulation of the Twist property with the following exceptions. Firstly, $C^{1}$-smoothness is only required for all $\left(u_{1}, u_{2}\right) \in \operatorname{int}\left(I_{E} \times I_{E} \backslash \Delta\right)$ such that $u_{1}$ nor $u_{2}$ is a critical point 8 . Secondly, when an equilibrium point $u_{*} \in I_{E}$ is a saddlefocus or a center then $\tau\left(u_{1}, u_{2}\right)$ is not continuous at $\left(u_{*}, u_{*}\right) \sqrt{9}$. In the case that $u_{1}$ and/or $u_{2}$ is an equilibrium point of real saddle type then $\tau$ can be $\propto$ 10. We refer

\footnotetext{
${ }^{7}$ Most of the results in this paper also hold for slightly weaker conditions. For example, when we do not require the family of solutions/extrema to be minimizers of $J_{E}\left(u_{1}, u_{2}\right)$ then we obtain the same results, the information on the index excluded. For the case where the family is continuous but not $C^{1}$ we refer to Section 2.3 .

${ }^{8}$ Singular energy levels connected components of $\pi^{u} N_{E}$ can have internal critical points. This will be discussed in Section 3.3 .

${ }^{9}$ It still holds that $J_{E}\left[u\left(t ; u_{1}, u_{2}\right)\right] \rightarrow 0$ as $\left(u_{1}, u_{2}\right) \rightarrow\left(u_{*}, u_{*}\right)$.

${ }^{10}$ We then consider $u$ on either $[0, \infty),(-\infty, 0]$ or $\mathbb{R}$ (whichever is appropriate) and require that $u\left(t ; u_{1}, u_{2}\right)$ converges on compact sets as $u_{1}$ and/or $u_{2}$ tends to the critical point.
} 
to Section 3.2 and Appendix $\mathrm{A}$ for more information on singular energy levels and equilibrium points.

Definition 3. A Lagrangian system $(L, d t)$ is called a Twist system on an interval component $I_{E}$ if both Hypotheses $(\mathrm{H})$ and $(\mathrm{T})$ are satisfied.

Using Hypothesis (T) we can derive the following regularity properties for $S$.

Lemma 4. Let $E$ be a regular value. If $(L, d t)$ is a Twist system on an interval component $I_{E}$, then the function $S_{E}\left(u_{1}, u_{2}\right)$ is of class $C^{2}\left(\operatorname{int}\left(I_{E} \times I_{E} \backslash \Delta\right)\right) \cap$ $C^{1}\left(I_{E} \times I_{E} \backslash \Delta\right) \cap C^{0}\left(I_{E} \times I_{E}\right)$.

Proof. Due to the smoothness assumption in (T) and the regularity of solutions of the Euler-Lagrange equations (see Section 1.1), we have that $u\left(t ; u_{1}, u_{2}\right)$ varies smoothly with $\left(u_{1}, u_{2}\right)$ with values in $C^{2}$. It is easily seen that $S_{E}\left(u_{1}, u_{2}\right)=$ $J_{E}\left[u\left(t ; u_{1}, u_{2}\right)\right]$ is a $C^{1}$-function on $I_{E} \times I_{E} \backslash \Delta$. Lemma 2 and Equation (3) show that $\partial_{1} S\left(u_{1}, u_{2}\right)=-p_{u_{1}}$ and $\partial_{2} S\left(u_{1}, u_{2}\right)=p_{u_{2}}$. It follows from the smoothness assumption in (T) and the fact that all solutions obey (2) that $p_{u_{1}}$ and $p_{u_{2}}$ are $C^{1}$ functions of $\left(u_{1}, u_{2}\right) \sqrt{11}$, hence $S_{E}$ is a $C^{2}$-function on $\operatorname{int}\left(I_{E} \times I_{E} \backslash \Delta\right)$. Continuity of $S_{E}$ at the diagonal follows either via simple squeezing estimates in the variational problem, or by analysing the shooting map 12 . This yields that $\left.S_{E}\right|_{\Delta} \equiv 0$.

If $S$ is considered on $I_{E}^{1} \times I_{E}^{2}$, where $I_{E}^{i}, i=1,2$, are different connected components of $\pi^{u} N_{E}$, then one does not expect $S_{E}$ to be defined on all of $I_{E}^{1} \times I_{E}^{2}$. The next lemma reveals some important properties of the generating function $S$. For the remainder of this section we assume that $E$ is a regular value and we consider interval components $I_{E}$ on which $(L, d t)$ is a Twist system.

Lemma 5. Let $E$ be a regular value. Then

(1) $\partial_{1} S\left(u_{1}, u_{2}\right)=-p_{u_{1}}$ and $\partial_{2} S\left(u_{1}, u_{2}\right)=p_{u_{2}}$ for all $\left(u_{1}, u_{2}\right) \in I_{E} \times I_{E} \backslash \Delta$,

(2) $\partial_{1} \partial_{2} S\left(u_{1}, u_{2}\right)>0$ for all $\left(u_{1}, u_{2}\right) \in \operatorname{int}\left(I_{E} \times I_{E} \backslash \Delta\right)$, and

(3) $\left.\partial_{n_{ \pm}} S\right|_{\text {int }(\Delta)}=+\infty$, where $n_{ \pm}=(\mp 1, \pm 1)^{T} \cdot 13$

Proof. Part (1) has been dealt with in the proof of Lemma 4. For part (2) of this lemma we argue as follows: $\partial_{1} \partial_{2} S\left(u_{1}, u_{2}\right)=\frac{\partial p_{u_{2}}}{\partial u_{1}}=-\frac{\partial p_{u_{1}}}{\partial u_{2}}$. Because of the uniqueness of the initial value problem for $x^{\prime}=X_{H}(x)$ the variable $-p_{u_{1}}$ is a strictly increasing function of $u_{2}$ ( $u_{1}$ fixed) ${ }^{14}$. In exactly the same way $p_{u_{2}}$ is a strictly increasing function of $u_{1}$ ( $u_{2}$ fixed). Therefore $\partial_{1} \partial_{2} S\left(u_{1}, u_{2}\right) \geq 0$. On the other hand using the smooth dependence on initial data for $x^{\prime}=X_{H}(x)$ and the smoothness of $\tau\left(u_{1}, u_{2}\right)$, it follows that both $u_{2}=u_{2}\left(u_{1}, p_{u_{1}}\right)$ and $u_{1}=u_{1}\left(u_{2}, p_{u_{2}}\right)$ are smooth functions. This implies that $\frac{\partial p_{u_{1}}}{\partial u_{2}} \neq 0$ and $\frac{\partial p_{u_{2}}}{\partial u_{1}} \neq 0$, and thus $\partial_{1} \partial_{2} S\left(u_{1}, u_{2}\right)>0$.

As for part (3) we only consider the derivative in the direction $n_{+}$(the other case is similar). We have that $u^{\prime \prime \prime}(0), u^{\prime \prime \prime}(\tau) \rightarrow-\infty$ as $u_{1} \rightarrow u_{2}$ since $u^{\prime \prime}(0), u^{\prime \prime}(\tau) \neq 0$

\footnotetext{
${ }^{11}$ At points $t \in(0, \tau)$ we have $p_{u}=\left(-\frac{\partial L}{\partial u^{\prime \prime}} u^{\prime \prime}+L+E\right) / u^{\prime}$ which depends smoothly on $\left(u_{1}, u_{2}\right)$ since $u^{\prime} \neq 0$ for $t \in(0, \tau)$. The smooth dependence of the initial value problem for the Hamiltonian flow now ensures that $p_{u}(0)$ and $p_{u}(\tau)$ depend smoothly on $\left(u_{1}, u_{2}\right)$ as well.

${ }^{12}$ In both cases the corner points $\partial \Delta$ have to be dealt with separately.

${ }^{13}$ This should be read as follows: when we approach a point $(\tilde{u}, \tilde{u}) \in \operatorname{int}(\Delta)$ from within the region $\left\{u_{2}>u_{1}\right\}$ then $\partial_{n_{+}} S \rightarrow \infty$ as $\left(u_{1}, u_{2}\right) \rightarrow(\tilde{u}, \tilde{u})$.

${ }^{14}$ It could also be strictly decreasing but this is excluded by part (3) of the lemma.
} 
on $\operatorname{int}(\Delta)$. For $p_{u}$ it holds that

$$
p_{u}=\partial_{v} L(u, 0, w)-\partial_{v w}^{2} L(u, 0, w) u^{\prime \prime}-\partial_{w}^{2} L(u, 0, w) u^{\prime \prime \prime}
$$

and thus $p_{u_{i}} \rightarrow \infty, i=1,2$.

The question of finding bounded characteristics for $(L, d t)$ can now best be formulated in terms of $S$. Extremizing the action $J_{E}$ over a space of 'broken geodesics' now corresponds to finding critical points of the formal sum $\sum_{n \in \mathbb{Z}} S\left(u_{n}, u_{n+1}\right)$. Formally we seek critical points (bounded sequences) of the infinite sum

$$
W\left(\cdots, u_{-1}, u_{0}, u_{1}, \cdots\right)=\sum_{i \in \mathbb{Z}} S\left(u_{i}, u_{i+1}\right)
$$

Since this sum is usually not well-defined for bounded sequences $\left(u_{i}\right)_{i \in \mathbb{Z}} \in \ell^{\infty}(\mathbb{Z})$, we say that a sequence is a critical sequence, or critical point of $W$, if

$$
\partial_{2} S\left(u_{i-1}, u_{i}\right)+\partial_{1} S\left(u_{i}, u_{i+1}\right)=0, \quad \forall i \in \mathbb{Z} .
$$

Such equations are called second order recurrence relations (see e.g. 6, 22 for related recurrence relations in the context of Twist diffeomorphisms). If (7) is satisfied for all $i \in \mathbb{Z}$, then $u$-laps can be glued to a $C^{3}$-function for which all derivatives up to order three match. Indeed, Equation (77) means that the third derivatives match 15 . Since every $u$-lap satisfies the Euler-Lagrange equations we then get a $C_{b}^{3}$-function $u$ that is stationary for $J[u]$. Of course, if we seek periodic sequences, i.e. sequences $\left(u_{i}\right)_{i \in \mathbb{Z}}$ with $u_{i+2 n}=u_{i}$, where $2 n$ is called the period, we may look for critical points of the restricted action $W_{2 n}=\sum_{i=1}^{2 n} S\left(u_{i}, u_{i+1}\right)$ defined on $I_{E}^{2 n} 16$ This corresponds to finding closed characteristics for $(L, d t)$. The period can be linked to various topological properties of $u$ and $x$ (in the Hamiltonian system $(H, \omega))$ such as knotting and linking of closed characteristics, see [12, 13]. Moreover, periodic sequences as critical points of $W_{2 n}$ have a Morse index, which is exactly the Morse index of a closed characteristic $u$ as a critical point of $\left.J\right|_{\Omega_{\text {per }}}$.

Lemma 6. Let $E$ be a regular value. Let $\boldsymbol{u}=\left(u_{i}\right)_{i \in \mathbb{Z}} \in \ell^{\infty}(\mathbb{Z}), u_{i} \in \operatorname{int}\left(I_{E}\right)$, be a periodic sequence with period $2 n$, which is a stationary point of $W_{2 n}$ with index $\mu(\boldsymbol{u}) \leq 2 n$. Then the associated closed characteristic $u$ for $(L, d t)$ is stationary for $J[u]$ and the Morse index of $u$ is also $\mu(\boldsymbol{u})$, and vice versa.

Proof. Let $\mathbf{u}$ be stationary for $W_{2 n}$, i.e. $d W_{2 n}(\mathbf{u})=0$. Concatenating the $u$-laps between the consecutive extrema $u_{i}$ yields a $\tau$-periodic $C^{3}$-function $u$ that satisfies the Euler-Lagrange equations of $(L, d t)$. It may be clear that the function $u$ is an extremal of (4). The statement concerning the Morse index $\mu(\mathbf{u})=\mu(u)$ can be proved as follows. The assumption that $u_{i} \in \operatorname{int}\left(I_{E}\right)$ implies that $u^{\prime \prime} \neq 0$ at extrema of $u(t)$. This implies that the number of monotone laps is conserved under small perturbations in $\Omega_{\text {per }}=\bigcup_{\tau>0} C^{2}\left(S^{1}, \tau\right)$.

A function $w$ in a small neighbourhood of $u$ can be characterized by the heights of the extrema $u_{1}, \ldots, u_{2 n}$ (cylic), the distances between the extrema $\tau_{1}, \ldots, \tau_{2 n}$,

\footnotetext{
${ }^{15}$ It holds that $\partial_{2} S\left(u_{i-1}, u_{i}\right)+\partial_{1} S\left(u_{i}, u_{i+1}\right)=\partial_{w}^{2} L\left(u_{i}, 0, u_{i}^{\prime \prime}\right)\left(-u_{i}^{\prime \prime \prime}+\tilde{u}_{i}^{\prime \prime \prime}\right)$, where $u_{i}^{\prime \prime \prime}$ is the third derivative on the left and $\tilde{u}_{i}^{\prime \prime \prime}$ is the third derivative on the right.

${ }^{16}$ The function $W_{2 n}$ is continuous on $I_{E}^{2 n}$ and is of class $C^{2}$ on the set $\left\{\left(u_{1}, \ldots, u_{2 n}\right) \in\right.$ $\left.\operatorname{int}\left(I_{E}^{2 n}\right) \mid u_{i} \neq u_{i+1}, \forall i=1, . ., 2 n\right\}$, with $u_{2 n+1} \equiv u_{1}$.
} 
and the deviations $v_{i}(t) \in C_{0}^{2}([0,1])$ of the minimizing laps, namely

$$
\begin{aligned}
& w(t)=u\left(\frac{\tau\left(u_{i}, u_{i+1}\right)}{\tau_{i}}\left(t-T_{i}\right) ; u_{i}, u_{i+1}\right)+v_{i}\left(\frac{1}{\tau_{i}}\left(t-T_{i}\right)\right) \\
& \quad \text { for all } t \in\left[T_{i}, T_{i+1}\right], i=1, \ldots, 2 n,
\end{aligned}
$$

where $u\left(t ; u_{i}, u_{i+1}\right)$ and $\tau\left(u_{i}, u_{i+1}\right)$ is the minimizing pair defined in hypothesis $(\mathrm{T})$, and $T_{i}=\sum_{k=1}^{i-1} \tau_{k}$. Consequently, $T_{u} \Omega_{\text {per }}$ can be identified with $\left(C_{0}^{2}([0,1]) \times \mathbb{R}\right)^{2 n} \oplus$ $\mathbb{R}^{2 n}$, separating the dependence on the heights of the extrema from the other contributions. The linear operator $d^{2} J_{E}[u]$ induces a linear operator on $\left(C_{0}^{2}([0,1]) \times \mathbb{R}\right)^{2 n}$, which is non-negative. Consequently the Morse index of $d^{2} J_{E}[u]$ is equal to the Morse index of the induced operator on $\mathbb{R}^{2 n}$. This induced operator is in fact $d^{2} W_{2 n}[\mathbf{u}]$ (for more details see e.g. [23]: case of broken geodesics).

For points $u_{1} \in \partial I_{E}$ additional information about $S$ can be obtained. Denote the left boundary point of $I_{E}$ by $u^{-}$and right boundary point by $u^{+}$.

Lemma 7. Let $E$ be a regular value. Let $u^{-} \in \partial I_{E}$ (assuming that there exists a left boundary point) then $\partial_{1} S\left(u^{-}, \tilde{u}\right)>-\partial_{v} L\left(u^{-}, 0,0\right)$ and $\partial_{2} S\left(\tilde{u}, u^{-}\right)>$ $\partial_{v} L\left(u^{-}, 0,0\right)$ for $\tilde{u}>u^{-}$. Similarly, if $u^{+} \in \partial I_{E}$ then $\partial_{1} S\left(u^{+}, \tilde{u}\right)<-\partial_{v} L\left(u^{+}, 0,0\right)$ and $\partial_{2} S\left(\tilde{u}, u^{+}\right)<\partial_{v} L\left(u^{+}, 0,0\right)$ for all $\tilde{u}<u^{+}$.

Proof. Let us prove the above inequalities for $\partial_{1} S$ as the case for $\partial_{2} S$ leads to an analogous argument. We start with the left boundary point $u^{-}$. We seek an increasing lap from $u^{-}$to $\tilde{u}$. At $u_{1}=u^{-}$it holds that $-L\left(u^{-}, 0,0\right)=E, u_{1}^{\prime \prime}=0$ and $\partial_{u} L\left(u^{-}, 0,0\right)>0$, which implies that $u_{1}^{\prime \prime \prime}(0)>0$. By contradiction, suppose that $u^{\prime}(0)=u^{\prime \prime}(0)=u^{\prime \prime \prime}(0)=0$. On one hand we have $p_{v}^{\prime}=\partial_{v} L-p_{u}$ and on the other hand $p_{v}^{\prime}=\partial_{u w}^{2} L u^{\prime}+\partial_{v w}^{2} L u^{\prime \prime}+\partial_{w}^{2} L u^{\prime \prime \prime}$. From the former and the Euler-Lagrange equation we see that $p_{v}^{\prime \prime}(0)=-\partial_{u} L(0)<0$, so that

$$
\lim _{\varepsilon \rightarrow 0} \frac{\left(\partial_{u w}^{2} L u^{\prime}+\partial_{v w}^{2} L u^{\prime \prime}+\partial_{w}^{2} L u^{\prime \prime \prime}\right)(\varepsilon)}{\varepsilon}=\lim _{\varepsilon \rightarrow 0} \frac{\partial_{w}^{2} L u^{\prime \prime \prime}(\varepsilon)}{\varepsilon}=-\partial_{u} L(0)<0 .
$$

We conclude (using condition $(\mathrm{H}))$ that $u^{\prime \prime \prime}(t)<0$ in a right neighborhood of 0 , which contradicts the fact that we are dealing with an increasing lap.

It now follows that $p_{u_{1}}=\partial_{v} L\left(u^{-}, 0,0\right)-\partial_{w}^{2} L\left(u^{-}, 0,0\right) u_{1}^{\prime \prime \prime}(0)<\partial_{v} L\left(u^{-}, 0,0\right)$. Therefore $\partial_{1} S\left(u^{-}, \tilde{u}\right)=-p_{u^{-}}>-\partial_{v} L\left(u^{-}, 0,0\right)$. For the right boundary point $u^{+}$ we obtain $\partial_{1} S\left(u^{+}, \tilde{u}\right)=-p_{u^{+}}<-\partial_{v} L\left(u^{+}, 0,0\right)$, since $u$ is a decreasing lap.

Since $S_{E}$ is only continuous at $\partial \Delta$ the gradient is not well-defined there. The following technical lemma states that near the corners $\partial \Delta$ the gradient $\nabla W_{2}$ points away from the corner into $I_{E} \times I_{E} \backslash \Delta$, which allows us to use an interior set of $I_{E} \times I_{E} \backslash \Delta$ preserving the boundary behavior as derived before (see Figure 2 ).

Lemma 8. Let $I_{E}=\left[u^{-}, u^{+}\right]$be a compact interval component such that neither $u^{-}$nor $u^{+}$is an equilibrium point. Then there exists a $\delta_{0}>0$ such that

(i) $\partial_{1} S\left(u_{-}, u_{-}+\delta\right)+\partial_{2} S\left(u_{-}+\delta, u_{-}\right)>0, \quad \partial_{2} S\left(u_{-}, u_{-}+\delta\right)+\partial_{1} S\left(u_{-}+\delta, u_{-}\right)>0$,

(ii) $\partial_{1} S\left(u_{+}-\delta, u_{+}\right)+\partial_{2} S\left(u_{+}, u_{+}-\delta\right)<0, \quad \partial_{2} S\left(u_{+}-\delta, u_{+}\right)+\partial_{1} S\left(u_{+}, u_{+}-\delta\right)<0$, for any $0<\delta \leq \delta_{0}$.

Proof. We prove part (i); the proof of part (ii) is completely analogous. The first statement in (i) follows immediately from Lemma [7. For the second statement in (i) we argue as follows. Choose $\delta>0$ sufficiently small. For an increasing lap $u(t), t \in[0, T]$, connecting $u_{-}$and $u_{-}+\delta$, it holds that $p_{v}^{\prime \prime}(0)<0$ (from the 


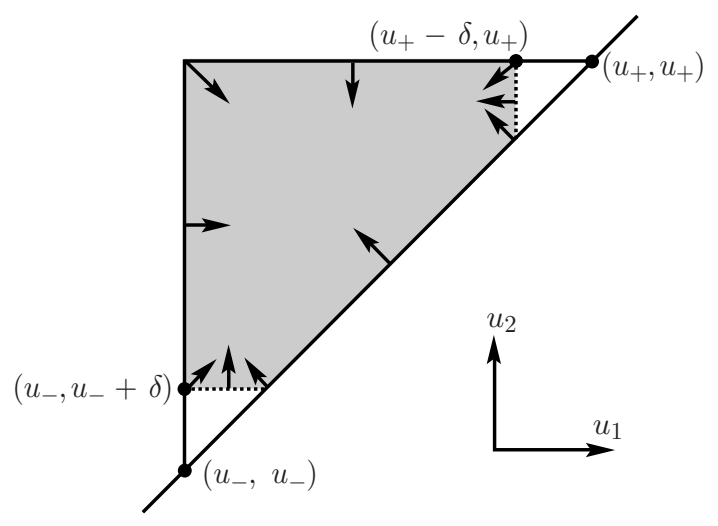

Figure 2. The behavior of $W_{2}$ near $\partial \Delta$. The transversality of $\partial W_{2}$ at $u_{1}=u_{+}-\delta$, and $u_{2}=u_{-}+\delta$ follows from the twist property.

Euler-Lagrange equation) and in fact $p_{v}^{\prime \prime}(t)<0$ on $[0, T]$ for small $\delta$ since $T \rightarrow 0$ as $\delta \rightarrow 0$. Moreover, $p_{v}^{\prime}(0)=\partial_{w}^{2} L u^{\prime \prime \prime}(0)>0$ as in the proof of Lemma 7 . The points $\left(u_{-}, p_{v}(0)\right)$ and $\left(u_{-}+\delta, p_{v}(T)\right)$ both belong to $N_{E}$. The set $N_{E}$ is a smooth closed curve in the $\left(u, p_{v}\right)$-plane whose projection onto the $u$-axis is $\left[u_{-}, u_{+}\right]$. At $\left(u_{-}, p_{v}(0)\right)$ the tangent to $N_{E}$ is vertical (since $\partial_{p_{v}} L^{*}\left(u_{-}, 0, p_{v}(0)\right)=u^{\prime \prime}(0)=0$ and $\left.\partial_{u} L^{*}\left(u_{-}, 0, p_{v}(0)\right)=\partial_{u} L\left(u_{-}, 0,0\right)<0\right)$. Because $u^{\prime \prime}(T)<0$ and $\delta$ is small, it follows that $p_{v}(T)<p_{v}(0)$. Therefore $p_{v}^{\prime}\left(t_{0}\right)<0$ for some intermediate point $t_{0} \in(0, T)$. The definite sign of $p_{v}^{\prime \prime}$ then implies that $p_{v}^{\prime}(T)<0$.

Now

$$
\begin{aligned}
\partial_{2} S\left(u_{-}, u_{-}+\delta\right) & =p_{u}(T)=\partial_{v} L\left(u_{-}+\delta, 0, u^{\prime \prime}(T)\right)-p_{v}^{\prime}(T) \\
& >\partial_{v} L\left(u_{-}+\delta, 0, u^{\prime \prime}(T)\right) .
\end{aligned}
$$

The same can be done for a decreasing lap $\tilde{u}(t), t \in[0, T]$, connecting $u_{-}+\delta$ and $u_{-}$, which gives $\partial_{1} S\left(u_{-}+\delta, u_{-}\right)=-\tilde{p}_{u}(0)=-\partial_{v} L\left(u_{-}+\delta, 0, \tilde{u}^{\prime \prime}(0)\right)+\tilde{p}_{v}^{\prime}(0)>$ $-\partial_{v} L\left(u_{-}+\delta, 0, \tilde{u}^{\prime \prime}(0)\right)$. This implies that

$$
\begin{aligned}
\partial_{2} S\left(u_{-}, u_{-}\right. & +\delta)+\partial_{1} S\left(u_{-}+\delta, u_{-}\right) \\
& >\partial_{v} L\left(u_{-}+\delta, 0, u^{\prime \prime}(T)\right)-\partial_{v} L\left(u_{-}+\delta, 0, \tilde{u}^{\prime \prime}(0)\right) .
\end{aligned}
$$

Noticing that $u^{\prime \prime}(T)=\tilde{u}^{\prime \prime}(0)$ finishes the proof.

2.2. Examples of Twist systems. An example of a class of Lagrangians for which we can verify the Twist property in various cases is given by $L\left(u, u^{\prime}, u^{\prime \prime}\right)=$ $\frac{1}{2}\left|u^{\prime \prime}\right|^{2}+K\left(u, u^{\prime}\right)$. Most of the fourth order equations coming from physical models are derived from Lagrangians of this form. We could tag such systems as fourth order mechanical systems based on the analogy with second order mechanical systems given by Lagrangians of the form $L\left(u, u^{\prime}\right)=\frac{1}{2}\left|u^{\prime}\right|^{2}+K(u)$ (integrable systems). The Lagrangian $L$ clearly satisfies Hypothesis $(\mathrm{H})$ and $(L, d t)$ is thus equivalent to the Hamiltonian system $(H, \omega)$ with $\omega$ the standard symplectic form on $\mathbb{R}^{4}$ (see Section (1) and $H(x)=p_{u} v+\frac{1}{2} p_{v}^{2}-K(u, v)$. For a regular energy value $E$ the set $\pi^{u} N_{E}$ is given by $\pi^{u} N_{E}=\{u \mid K(u, 0)+E \geq 0\}$. If $E$ is regular it holds that $K(u, 0)+E>0$ for $u \in \operatorname{int}\left(I_{E}\right)$, and $K(u, 0)+E=0$ for $u \in \partial I_{E}$. 
Lemma 9. Let $I_{E}$ be a connected component of $\pi^{u} N_{E}$ (E not necessarily regulat $\left.{ }^{17}\right)$. Assume that

(a) $\frac{\partial K}{\partial v} v-K(u, v)-E \leq 0$ for all $u \in I_{E}$ and $v \in \mathbb{R}$,

(b) $\frac{\partial^{2} K}{\partial v^{2}}|v|^{2}-\frac{5}{2}\left\{\frac{\partial K}{\partial v} v-K(u, v)-E\right\} \geq 0$ for all $u \in I_{E}$ and $v \in \mathbb{R}$.

Then for any pair $\left(u_{1}, u_{2}\right) \in I_{E} \times I_{E} \backslash \Delta$ Problem (6) has a unique minimizer $(u, \tau) \in$ $X_{\tau} \times \mathbb{R}^{+}$(in fact the only critical point), and the minimizer $u\left(t ; u_{1}, u_{2}\right)$ depends $C^{1}$-smoothly on $\left(u_{1}, u_{2}\right)$ for $\left(u_{1}, u_{2}\right) \in \operatorname{int}\left(I_{E} \times I_{E} \backslash \Delta\right)$ 18.

For the proof of this lemma we refer to Appendix B.

At this point we are not able to prove that the Twist property holds for more general systems under some mild growth conditions on $K$ without assuming (a) and (b). However, numerical experiments (see Section 4.1) for various Lagrangians suggest that Lemma 9 is still valid, although we do not have a proof of this fact. Milder conditions on $K$ sometimes only allow the existence of a continuous family $u\left(t ; u_{1}, u_{2}\right)$. We come back to this case in Section 2.3. The conditions given in Lemma 9 already allow for a large variety of Lagrangians that occur in various physical models. We will give a few examples of such systems now.

2.2.1. The eFK/Swift-Hohenberg system. The eFK/Swift-Hohenberg Lagrangian is given by $L\left(u, u^{\prime}, u^{\prime \prime}\right)=\frac{1}{2}\left|u^{\prime \prime}\right|^{2}+\frac{\alpha}{2}\left|u^{\prime}\right|^{2}+F(u)$, where $\alpha \in \mathbb{R}$ and $F$ is a smooth potential function. The Hamiltonian in this case is $H(x)=p_{u} v+\frac{1}{2} p_{v}^{2}-\frac{\alpha}{2} v^{2}-F(u)$. Connected components of $\pi^{u} N_{E}$ are sets of the form $\{u \mid F(u)+E \geq 0\}$.

In the case that $\alpha>0$ this $L$ is referred to as the eFK-Lagrangian (see e.g. [15, 17, 18]), and in the case $\alpha \leq 0$ it is usually referred to as the Swift-Hohenberg Lagrangian 24, 31 32. For example, $F(u)=\frac{1}{4}\left(u^{2}-1\right)^{2}$ is the classical eFK/SwiftHohenberg potential [28, 29], $F(u)=\frac{1}{3} u^{3}-\frac{1}{2} u^{2}$ gives the water-wave model [9], $F(u)=-\frac{1}{4}\left(u^{2}-1\right)^{2}$ is the potential of a nonlinear optics model [1].

If $\alpha \leq 0$ then the conditions (a) and (b) are satisfied for any interval component $I_{E}$. The Swift-Hohenberg system is therefore a Twist system for all interval components. For $\alpha>0$ this is not immediately clear (conditions (a) and (b) are not satisfied)19. More details on eFK/Swift-Hohenberg systems are given in Section 3.4.

2.2.2. The suspension-bridge model. The suspension bridge model is a special case of the Swift-Hohenberg equation, namely $L\left(u, u^{\prime}, u^{\prime \prime}\right)=\frac{1}{2}\left|u^{\prime \prime}\right|^{2}-\frac{c^{2}}{2}\left|u^{\prime}\right|^{2}+F(u)$, with $F(u)=e^{u}-u-1$ (see [30]). Clearly, the suspension bridge model is a Twist system for all $c \in \mathbb{R}$. For more details see Section 3.4 This model is particularly intriguing due to the specific form of the potential function $F$. The growth of $F$ for $u \rightarrow \infty$ is essentially different from the growth for $u \rightarrow-\infty$ which has far reaching consequences for the set of closed characteristics.

2.2.3. The fifth order $K d V$ equation. Consider $L\left(u, u^{\prime}, u^{\prime \prime}\right)=\frac{1}{2}\left|u^{\prime \prime}\right|^{2}+K\left(u, u^{\prime}\right)$, where $K\left(u, u^{\prime}\right)=\frac{1}{2}(\alpha+2 \mu u)\left|u^{\prime}\right|^{2}+F(u)$ with $F(u)=\frac{\kappa}{3} u^{3}+\frac{\sigma}{2} u^{2}$, which describes traveling waves in a fifth order Korteweg-de Vries equation (see e.g. [26]). In

\footnotetext{
${ }^{17}$ If $E$ is a singular energy level, then we require the critical points to be non-degenerate.

${ }^{18}$ If $E$ is a singular energy level, then $C^{1}$-regularity holds for all $\left(u_{1}, u_{2}\right) \in \operatorname{int}\left(I_{E} \times I_{E} \backslash \Delta\right)$ for which neither $u_{1}$ nor $u_{2}$ is a critical point.

${ }^{19} \mathrm{~J}$. Kwapisz 20] proves that the $\left(C^{0}\right)$ Twist property $\left(\mathrm{T}^{\prime}\right)$ (see Section 2.3) is satisfied for the eFK Lagrangian $(\alpha>0)$ on interval components $I_{E}$ for which $F(u)+E$ has at most one internal extremum (a maximum).
} 
order for the theory to be applicable the conditions on $K$ in Lemma 9 imply that $\alpha+2 \mu u \leq 0$ for $u \in I_{E}$. The case $\mu=0$ is the Swift-Hohenberg equation again. Let us assume for example that $\kappa, \sigma>0$, then one finds compact intervals $I_{E}$ for values $-\frac{\sigma^{3}}{6 \kappa^{2}}<E \leq 0$. These intervals are contained in $\left[-\frac{3 \sigma}{2 \kappa}, 0\right]$. For $\mu>0$ the condition becomes $u<-\frac{\alpha}{2 \mu}$, which is for instance satisfied for all $u \in I_{E}$ if $\alpha<0$. For $\mu<0$ the condition becomes $u>-\frac{\alpha}{2 \mu}$, which is satisfied for all $u \in I_{E}$ if $\alpha<2 \frac{\sigma \mu}{\kappa}$. Many more combinations can be found by also varying the signs of $\kappa$ and $\sigma$.

2.3. The $C^{0}$-Twist property. As we already remarked before, the theory developed in this paper can be adjusted for $C^{1}$-generating functions. We will point out the difficulties and how the theory has to be adjusted at the end of this section. First we start with a weaker version of the Twist property that ensures the existence of $C^{1}$-generating functions.

$\left(\mathrm{T}^{\prime}\right) \inf \left\{J_{E}[u] \mid u \in X_{\tau}\left(u_{1}, u_{2}\right), \tau \in \mathbb{R}^{+}\right\}$has a minimiser $u\left(t ; u_{1}, u_{2}\right)$ for all $\left(u_{1}, u_{2}\right)$ $\in I_{E} \times I_{E} \backslash \Delta$, and $u$ and $\tau$ are continuous functions of $\left(u_{1}, u_{2}\right)$.

Hypothesis $\left(\mathrm{T}^{\prime}\right)$ is often easier to verify than the stronger Hypothesis $(\mathrm{T})$. Let $I_{E}$ be an interval component and $(L, d t)$ is a Twist system on $I_{E}$ with respect to Hypothesis $\left(\mathrm{T}^{\prime}\right)$. Then $p_{u_{2}}\left(u_{1}, u_{2}\right)$ is strictly increasing in $u_{1}$ and $-p_{u_{2}}\left(u_{1}, u_{2}\right)$ is strictly increasing in $u_{2}$, and both are continuous in $\left(u_{1}, u_{2}\right)$. The maps $T_{ \pm}$as described in Section 1.2 are therefore monotone $\left(C^{1_{-}}\right)$Twist maps, which have a $C^{1}$-generating function $S_{E}\left(u_{1}, u_{2}\right)=J_{E}\left[u\left(t ; u_{1}, u_{2}\right)\right]$.

Lemma 10. Let $I_{E}$ be an interval component. If $(L, d t)$ is a Twist system with respect to Hypothesis $\left(T^{\prime}\right)$, then $S_{E}$ is a $C^{1}$-generating function on $I_{E} \times I_{E} \backslash \Delta$.

Property (2) of Lemma 5 is now replaced by the property that $\partial_{1} S$ and $\partial_{2} S$ are increasing functions of $u_{1}$ and $u_{2}$ respectively. The difficulties in working with $C^{1}$-generating functions are the definition of the Morse index and the gradient flow of $W=\sum_{i} S\left(u_{i}, u_{i+1}\right)$. In Section 3 we use the gradient flow of $W$ to find other critical points besides minima and maxima. One way of dealing with this problem is to approximate $S$ by $C^{2}$-functions. A $C^{1}$-Morse/Conley index can then be defined (see for instance [7, 8]). An analogue of Lemma 6] can also be proved now. Other properties of $S$ that we use in this paper, such as construction of isolating neighborhoods, do not need the $C^{2}$-regularity. For this reason we will continue with $C^{2}$-function keeping in mind that all result carry over to the $C^{1}$-case.

\section{EXISTENCE}

3.1. Simple closed characteristics for compact sections $N_{E}$. The properties of $S$ listed in Section 2.1 can be used to derive an existence result for simple closed characteristics. Before stating the theorem we need to introduce some additional notation: $I_{E} \times I_{E} \backslash \Delta=D_{E}^{+} \cup D_{E}^{-}$, where $D_{E}^{+}=\left\{\left(u_{1}, u_{2}\right) \in I_{E} \times I_{E} \backslash \Delta \mid u_{2}>u_{1}\right\}$, and $D_{E}^{-}$is defined analogously. The function $W_{2}\left(u_{1}, u_{2}\right)=S\left(u_{1}, u_{2}\right)+S\left(u_{2}, u_{1}\right)$ is a $C^{2}$-function on $\operatorname{int}\left(I_{E} \times I_{E} \backslash \Delta\right)$. Since $W_{2}\left(u_{1}, u_{2}\right)=W_{2}\left(u_{2}, u_{1}\right)$ we can restrict our analysis to $D_{E}^{+}$.

Throughout this section we again assume that $E$ is regular and $(L, d t)$ is a Twist system on $I_{E}$. 


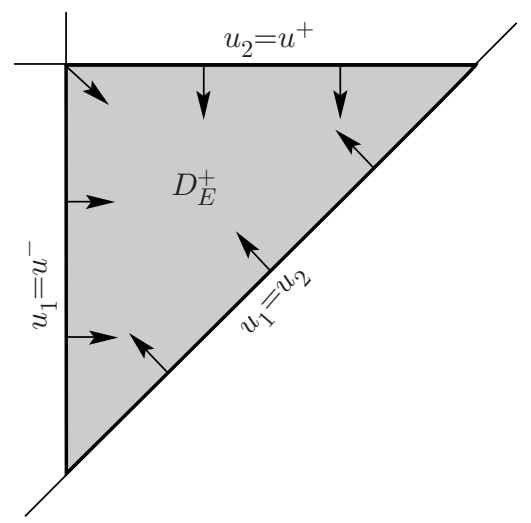

Figure 3. A picture of $D_{E}^{+}$. The arrows denote the direction of the gradient $\nabla W_{2}$ schematically (of course the gradient is not perpendicular to the boundary everywhere). Clearly the maximum of $W_{2}$ is attained in the interior of $D_{E}^{+}$.

Lemma 11. Assume that $\pi^{u} N_{E}$ contains a compact interval component $I_{E}$. Then $W_{2}$ has at least one maximum on $D_{E}^{+20}$.

Proof. We have that $\left.W_{2}\right|_{\Delta}=0$ and $W_{2}$ is strictly positive near $\operatorname{int}(\Delta)$ by Lemma[5 part (3). In fact the gradient $\nabla W_{2}$ points away from $\Delta$ near the diagonal and also points away from $\partial \Delta$ near the corner points (Lemma 8 ). Following Figure 2 we consider the region $D_{\delta} \subset D_{E}^{+}$defined by $D_{\delta}=\left\{\left(u_{1}, u_{2}\right) \in D_{E}^{+} \mid u_{2} \geq u_{-}+\delta, u_{1} \leq\right.$ $\left.u_{+}-\delta\right\}$. Since the set $\overline{D_{\delta}}$ is compact, $W_{2}$ must attain a maximum on set $\overline{D_{\delta}}$. It is immediately clear that $\max _{\left(u_{1}, u_{2}\right) \in \overline{D_{\delta}}} W_{2}\left(u_{1}, u_{2}\right)>0$.

Denote by $n_{1}=(1,0)^{T}$ the inward pointing normal on the left boundary $B_{1}=$ $\left\{\left(u^{-}, u_{2}\right) \mid u_{2} \in I_{E}\right\} \cap D_{\delta}$ and by $n_{2}=(0,-1)^{T}$ the inward pointing normal on $B_{2}=\left\{\left(u_{1}, u^{+}\right) \mid u_{1} \in I_{E}\right\} \cap D_{\delta}$. Using Lemma 7 we can now compute $\frac{\partial W_{2}}{\partial n_{1}}$ and $\frac{\partial W_{2}}{\partial n_{2}}$. For example let $u_{1}=u^{-}$, then $\frac{\partial W_{2}}{\partial n_{1}}=\partial_{1} S\left(u^{-}, u_{2}\right)+\partial_{2} S\left(u_{2}, u^{-}\right)>-\partial_{v} L\left(u^{-}, 0,0\right)+$ $\partial_{v} L\left(u^{-}, 0,0\right)=0$. Similarly, using Lemma 7 we derive that $\left.\frac{\partial W_{2}}{\partial n_{2}}\right|_{B_{2}}>0$. It follows from Lemma 8 that $\nabla W_{2}$ is also pointing inward at the boundaries $\left\{u_{1}=u_{+}-\delta\right\}$ and $\left\{u_{2}=u_{-}+\delta\right\}$ (see Figure 2). Summarizing, $\nabla W_{2}$ is pointing inward at $\partial D_{\delta}$, and $\left.W_{2}\right|_{\Delta}=0$. Therefore the maximum is attained $\operatorname{in} \operatorname{int}\left(D_{\delta}\right)$ (see also Figure 3).

If we study $W_{2 n}, n>1$, we do not necessarily find new closed characteristics for $(L, d t)$, i.e. critical points of $W_{2 n}$ of higher index may be the same closed characteristic traversed more than once. In the following sections we will describe some mechanisms that yield more geometrically distinct closed characteristics.

The above lemma can be slightly rephrased for Lagrangian systems (see Lemma 6). We do not have information about the nullity of $d^{2} J_{E}\left(u_{1}, u_{2}\right)$, so that

\footnotetext{
${ }^{20}$ From straightforward Morse theory for $W_{2}$ on $D_{E}^{+}$we obtain in addition that $b_{0} \geq 0, b_{1}-b_{0} \geq$ 0 and $b_{2}-b_{1}+b_{0}=1$, where $b_{i}$ is the number of critical points of index $i$ (in the case that $W_{2}$ is a Morse function).
} 
the large Morse index 21 of the solutions may be greater than 2, but the Morse index is certainly smaller than or equal to 2 .

Theorem 12. Assume that $\pi^{u} N_{E}$ contains a compact interval component $I_{E}$. Then $(L, d t)$ contains at least one simple closed characteristic $u(t) \in \operatorname{int}\left(I_{E}\right)$ with large Morse index greater than or equal to 2 and Morse index less than or equal to 2.

Theorem 12 states that the associated Hamiltonian system $(H, \omega)$ has at least one closed characteristic on $M_{E}$. Using the theory of this paper Theorem 12 is proved without the Twist property in [16]. The above theorem is reminiscent of first order Lagrangian systems: $L\left(u, u^{\prime}\right)$ with Euler-Lagrange equation $\frac{\partial L}{\partial u}-\frac{d}{d t} \frac{\partial L}{\partial u^{\prime}}=0$. Such systems may be labeled as mechanical systems if $\partial_{u^{\prime}} L>0$. On the compact components of $\left\{\left(u, u^{\prime}\right) \mid \frac{\partial L}{\partial u^{\prime}} u^{\prime}-L\left(u, u^{\prime}\right)=E\right\}$ closed characteristics exist (integrable system).

If $L$ is invariant with respect to the symmetry $t \mapsto-t$ it holds that $L(u, v, w)$ $=L(u,-v, w)$ for all $(u, v, w) \in \mathbb{R}^{3}$. A consequence of this symmetry is that $S\left(u_{1}, u_{2}\right)=S\left(u_{2}, u_{1}\right)$ which implies that we can study just $S$ (instead of $\left.W_{2}\right)$ to find simple closed characteristics in this case. Moreover, this symmetry of $L$ carries over to the simple closed characteristic: $u(t)$ is symmetric with respect to its extrema. Some Lagrangian systems are also invariant under the symmetry $u \mapsto-u$ which yields the relation $L(u, v, w)=L(-u,-v,-w)$. If $0 \in \pi^{u} N_{E}$ then there is at least one closed characteristic on the anti-diagonal $u_{1}=-u_{2}$. If the global maximum of $W_{2}$ is not on the anti-diagonal $u_{1}=-u_{2}$, then there are at least 2 more closed characteristics (by symmetry).

If we consider non-compact interval components $I_{E}$ there is no topological restriction that forces the existence of closed characteristics, and there need not exist any. In order to deal with this case (in forthcoming sections) more information about $L$ is needed: asymptotic behavior (see Section 3.4 ).

3.2. Singular energy levels. If $E$ is a singular energy level then there exist points $u \in \pi^{u} N_{E}$ for which $\partial_{u} L(u, 0,0)=0$ and $L(u, 0,0)+E=0$. For a singular value $E$ the connected components of $N_{E}$ are either smooth manifolds ( $\mathbb{R}$ or $S^{1}$ ), or they are characterized as: $N_{E}^{c} \simeq(\mathbb{R} \vee) S^{1} \vee \cdots \vee S^{1}(\vee \mathbb{R})$. The points in $\mathbb{R}^{2}$ on which $N_{E}$ fails to be a manifold lie on the $u$-axis, and are exactly the points $u$ for which $\partial_{u} L(u, 0,0)=0$ and $L(u, 0,0)+E=0$. The set of such points - critical points - is denoted by $\mathcal{C}\left(I_{E}\right)$. As before, $\pi^{u} N_{E}$ is a union of closed intervals. An interval component $I_{E}$ is defined as a subset of $\pi^{u} N_{E}$ such that $L(u, 0,0)+E>0$ for all $u \in \operatorname{int}\left(I_{E}\right)$ and $L(u, 0,0)+E=0$ for $u \in \partial I_{E}$. Since $E$ is singular two interval components $I_{E}^{1}$ and $I_{E}^{2}$ may have non-empty intersection, i.e. $I_{E}^{1} \cap$ $I_{E}^{2}=\{$ one point $\} \subset \mathcal{C}\left(I_{E}\right)$. Concatenations of interval components are discussed in Section 3.3. If we consider interval components with critical points geometric properties come into play. We assume that $(L, d t)$ is a Twist system for the interval components that we consider.

Let $I_{E}$ be an interval component for which $u^{-} \in \partial I_{E}$ is a critical point. In order to prove the analogue of Lemma 7 we need to know whether $u^{\prime \prime \prime}(0)$ is zero or not. This is determined by $\tau\left(u^{-}, u_{2}\right)$, i.e. if $\tau\left(u^{-}, u_{2}\right)<\infty$ then $u^{\prime \prime \prime}(0)>0$ (assuming $u_{2}>u^{-}$), and if $\tau\left(u^{-}, u_{2}\right)=\infty$ then $u^{\prime \prime \prime}(-\infty)=0$ (in the case that $\tau=\infty$ we consider $u$ on $[-\tau, 0]$ using translation invariance). These two cases can be distinguished by studying the singularity at $u^{-}$. We can compute the spectrum

${ }^{21}$ The large Morse index is defined as the sum of the Morse index and the nullity. 
of $u^{-}$which we will denote by $\sigma\left(u^{-}\right)$. We assume that we are dealing with nondegenerate singular points, i.e. $0 \notin \sigma\left(u^{-}\right)$. Critical points on the boundary of interval components obey $\partial_{u}^{2} L(u, 0,0)>0$. It is shown in Appendix $\mathrm{A}$ that there are three possible behaviors for $\sigma\left(u^{-}\right): \sigma\left(u^{-}\right) \subset \mathbb{R}, \sigma\left(u^{-}\right) \subset i \mathbb{R}$, or $\sigma\left(u^{-}\right) \subset \mathbb{C} \backslash\{\mathbb{R} \cup i \mathbb{R}\}$. In the latter case there is one eigenvalue in each quadrant. The three possible behaviors are categorized as real saddle, center and saddle-focus respectively. If $\sigma\left(u^{-}\right) \subset \mathbb{C} \backslash \mathbb{R}$ (center or saddle-focus), then $\tau\left(u^{-}, u_{2}\right)<\infty$ for all $u_{2} \in I_{E}$. It is immediately clear that Theorem 12 is still valid in that case. Also, if both $u^{+}$and $u^{-}$are critical points and have their spectrum in $\mathbb{C} \backslash \mathbb{R}$, Theorem 12 remains true.

Theorem 13. Let $E$ be a singular value and assume that $\sigma\left(\mathcal{C}\left(I_{E}\right)\right) \subset \mathbb{C} \backslash \mathbb{R}$. Then $(L, d t)$ contains at least one simple closed characteristic $u(t) \in \operatorname{int}\left(I_{E}\right)$ with large Morse index greater than or equal to 2 and Morse index less than or equal to 2.

The way to attack the problem of finding closed characteristics at singular energy levels in general is to again consider the function $W_{2}\left(u_{1}, u_{2}\right)=S\left(u_{1}, u_{2}\right)+S\left(u_{2}, u_{1}\right)$. Since $\left.W_{2}\right|_{\Delta} \equiv 0$ and strictly positive near $\Delta, W_{2}$ attains its global maximum in $I_{E} \times I_{E} \backslash \Delta$. As was already pointed out before, the maximum is attained in the interior of $I_{E} \times I_{E} \backslash \Delta$ if there are no critical points of $L(u, 0,0)$ in $\partial I_{E}$, or if critical points of $L(u, 0,0)$ have complex spectrum (Theorem 13). Thus in order for $W_{2}$ to attain its global maximum on the boundary, the interval component $\partial I_{E}$ needs to contain at least one critical point of $L(u, 0,0)$ with real spectrum.

The next question is: suppose $W_{2}$ attains its maximum at $\partial\left(I_{E} \times I_{E} \backslash \Delta\right)$, does this maximum correspond to a simple closed trajectory for $(L, d t)$ ? Again from the previous we know that at a point $\left(u_{1}, u_{2}\right) \in \partial\left(I_{E} \times I_{E} \backslash \Delta\right)$ it holds that $\partial_{1} W_{2} \geq 0$ if $u_{1}=u^{-}$and $\partial_{2} W_{2} \leq 0$ if $u_{2}=u^{+}$. A boundary maximum for which $u_{1}=$ $u^{-}$and $u_{2}=u^{+}$is called a co-dimension 2 point, and the remaining boundary points are called co-dimension 1 points. It is clear that at a co-dimension 1 point, for example at $u_{1}=u^{-}$, it holds that $\partial_{2} W_{2}=0$. Since we are assuming that this point is a maximum, and because $\partial_{1} W_{2} \geq 0$ it follows that the maximum is in fact a critical point. The same holds for a co-dimension 1 point at $u_{2}=$ $u^{+}$. Such points correspond to solutions $u(t)$ for which $u^{\prime \prime \prime}(-\infty)=u^{\prime \prime \prime}(\infty)=0$, and $u(-\infty)=u(\infty)=u^{-}$, and $u(t)$ is thus a homoclinic orbit. By the same reasoning co-dimension 2 points are also critical points. Such a point corresponds to a heteroclinic loop (two heteroclinic connections that form a loop).

Summarizing, we can introduce the notion of closed characteristic in the broad sense of the word: a simple closed periodic orbit, a simple homoclinic loop, or a simple heteroclinic loop (they all form a simple closed loop in the configuration plane). If we use this definition we obtain the following theorem.

Theorem 14. Assume that $\pi^{u} N_{E}$ has a compact interval component $I_{E}$ then $(L, d t)$ has at least one simple closed characteristic in the broad sense.

It is clear from the previous that a necessary condition for $(L, d t)$ to have a simple homoclinic loop to $u^{-}$is that $u^{-}$is a critical point of $L(u, 0,0)$ that has real spectrum (real saddle). The same holds for $u^{+}$. A necessary condition to find a simple heteroclinic loop between $u^{-}$and $u^{+}$is that both $u^{-}$and $u^{+}$are real saddles. Unfortunately, these conditions need not be sufficient 22 .

\footnotetext{
${ }^{22}$ For the eFK Lagrangian with $F(u)=\frac{1}{4}\left(u^{2}-1\right)^{2}$ it has been shown that the simple closed characteristic found in Theorem 14 corresponds to a heteroclinic loop if and only if the equilibrium points are real saddles [28, 33, 19].
} 
One way to guarantee the existence of a simple homoclinic loop to $u^{-} \in \mathcal{C}\left(I_{E}\right)$ is that $\tau\left(u^{-}, u_{2}\right)=\tau\left(u_{2}, u^{-}\right)=\infty$ for all $u_{2} \in I_{E} \sqrt{23}$, and either $u^{+} \notin \mathcal{C}\left(I_{E}\right)$ or $u^{+}$has complex spectrum. In that case $\partial_{1} S\left(u^{-}, u_{2}\right)=-\partial_{v} L\left(u^{-}, 0,0\right)$ for all $u_{2} \in I_{E}$. In terms of $W_{2}$ this yields that $\partial_{1} W_{2}\left(u^{-}, u_{2}\right)=0$ for all $u_{2} \in I_{E}$. We can now restrict $W_{2}$ to the line-segment $\left\{u_{1}=u^{-}\right\} \times I_{E}$. Define $W_{1}(u)=$ $\left.W_{2}\right|_{\left\{u_{1}=u^{-}\right\} \times I_{E}}=S\left(u^{-}, u\right)+S\left(u, u^{-}\right)$. It easily follows that (compare Lemma 7) $W_{1}\left(u^{-}\right)=0, W_{1}\left(u^{-}+\varepsilon\right)>0$ for $\epsilon>0$ sufficiently smal 24 and $W_{1}^{\prime}\left(u^{+}\right)<0$, and thus $W_{1}$ has at least one global maximum $u_{*}$ on $\left(u^{-}, u^{+}\right)$. The point $u_{*}$ corresponds to a homoclinic orbit to $u=u^{-}$.

Regarding the Morse index of this point/orbit we note the following. If $u_{*}$ is a (local) maximum of $W_{2}$ on $D_{E}^{+}$then the large Morse index is again equal to 2 . The corresponding homoclinic orbit has large Morse index greater than or equal to 2 and Morse index less than or equal to 2. However, restricted to the class of functions that are homoclinic to $u^{-}$it has large Morse greater than or equal to 1 and Morse index less than or equal to 1 (mountain-pass critical point)25.

3.3. Concatenation of interval components. Up to this point we have only considered single interval components $I_{E}$. When $E$ is a singular value then two interval components $I_{E}^{1}$ and $I_{E}^{2}$ may have a common boundary point. This boundary point is then necessarily a critical point. The concatenation of the interval components $I_{E}^{i}, i=1,2$, will be denoted by $I_{E}^{\#}$, and the critical point in $I_{E}^{1} \cap I_{E}^{2}$ is denoted by $u_{*}$. If $(L, d t)$ is a Twist system on both interval components $I_{E}^{1}$ and $I_{E}^{2}$ it does does not necessarily mean that $(L, d t)$ is a Twist system on the concatenated interval $I_{E}^{\#}$. One can easily give examples where $(L, d t)$ fails to satisfy the Twist property on $I_{E}^{\# 26}$ However, if $(L, d t)$ is Twist system on $I_{E}^{\#}$, and this is indeed true in many cases, more solutions can be found. In order to study this case we will use the gradient flow of $W_{2}$ :

$$
\begin{aligned}
& u_{1}^{\prime}=\partial_{2} S\left(u_{2}, u_{1}\right)+\partial_{1} S\left(u_{1}, u_{2}\right), \\
& u_{2}^{\prime}=\partial_{2} S\left(u_{1}, u_{2}\right)+\partial_{1} S\left(u_{2}, u_{1}\right), \quad u_{i} \in \operatorname{int}\left(I_{E}\right), \forall i=1,2
\end{aligned}
$$

with $u_{i} \in \operatorname{int}\left(I_{E}^{\#}\right)$ for $i=1,2$.

As before we can restrict our analysis to $D_{E}^{+}$. Define $D_{E, 1}^{+}=\left\{\left(u_{1}, u_{2}\right) \in I_{E}^{1} \times\right.$ $\left.I_{E}^{1} \mid u_{2}>u_{1}\right\}, D_{E, 2}^{+}=\left\{\left(u_{1}, u_{2}\right) \in I_{E}^{2} \times I_{E}^{2} \mid u_{2}>u_{1}\right\}$, and $D_{E, 3}^{+}=I_{E}^{1} \times I_{E}^{2} \backslash\left(u_{*}, u_{*}\right)$. On the domains $D_{E, 1}^{+}$and $D_{E, 2}^{+}$one can again apply Theorem 14 which yields the existence of maxima on each of these components. Note that this is independent of the type of $u_{*}$ (spectrum $\left.\sigma\left(u_{*}\right)\right)$. The following theorem will crucially use the fact that $u_{*}$ is a critical point for which $\sigma\left(u_{*}\right) \subset \mathbb{C} \backslash\{\mathbb{R} \cup i \mathbb{R}\}$, i.e. a saddle-focus.

\footnotetext{
${ }^{23}$ It follows from Lemma 5 part (2) that it in fact suffices that $\tau\left(u^{-}, u^{+}\right)=\tau\left(u^{+}, u^{-}\right)=\infty$.

${ }^{24}$ It follows from the linearization around $u^{-}$that $p_{u_{2}}<0$ for $u^{-}<u_{2}<u^{-}+\varepsilon$ when $\varepsilon$ is small enough.

${ }^{25}$ For the eFK Lagrangian with $F_{a}(u)=\int_{1}^{u}\left(s^{2}-1\right)(s-a) d s, 0 \leq a<1$, and $\alpha \geq 2 \sqrt{2(1-a)}$ the Twist property is satisfied on the interval component $I_{0}=\left[u^{-}, 1\right]$ and $\tau\left(u^{-}, 1\right)=\infty$. Therefore there exists a homoclinic loop in this case. The existence of such solutions for this problem was first proved in 27] by means of a different method. If the case $a=0$ is considered one obtains a heteroclinic loop (see e.g. [17]).

${ }^{26}$ For example consider the eFK Lagrangian with $F(u)=\frac{1}{4}\left(u^{2}-1\right)^{2}$. Take $E=0$, then $\pi^{u} N_{0}=\mathbb{R}$ is the concatenation of three intervals. If $\alpha \geq 2 \sqrt{2}$ then $(L, d t)$ is not a Twist system on $I_{0}^{\#}=\mathbb{R}$. However for $\alpha \leq 0$ the Twist property is satisfied on $\mathbb{R}$, and numerical experiments indicate the same for $2 \sqrt{2}>\alpha>0$. This is related to the behavior of the singularities $u= \pm 1$ (see Section 4.1).
} 


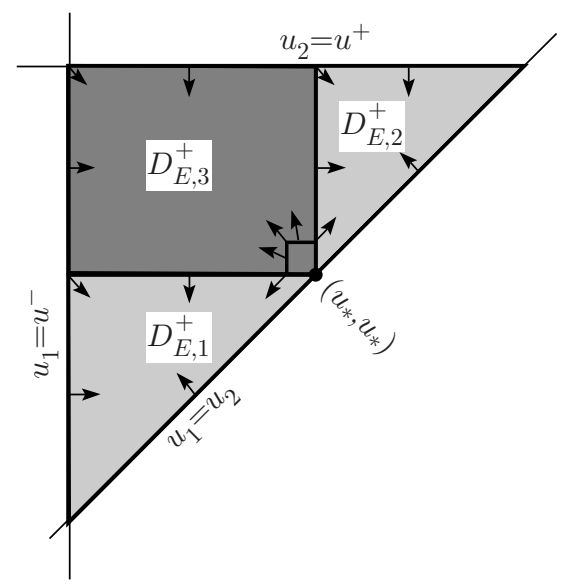

Figure 4 . The triangle $D_{E}^{+}$when a connected component of $\pi^{u} N_{E}$ consists of two compact interval components. The arrows denote (schematically) the direction of the gradient $\nabla W_{2}$. Clearly $W_{2}$ has maximum in $D_{E, 1}^{+}$and $D_{E, 2}^{+}$and a saddle point in $D_{E, 3}^{+}$.

Lemma 15. Let $I_{E}^{\#}$ be a concatenation of two compact interval components $I_{E}^{1}$ and $I_{E}^{2}$ and assume that the critical point $u_{*} \in I_{E}^{1} \cap I_{E}^{2}$ is a saddle-focus. Then $W_{2}$ has at least one maximum on each of the components $D_{E, i}^{+}, i=1,2$, and $W_{2}$ has a saddle point (critical point with large Morse index equal to 1 ) on the component $D_{E, 3}^{+}$.

Proof. The existence of at least one maximum on each of the components $D_{E, i}^{+}, i=$ 1,2 , follows directly from Theorem 14 As for the existence of saddle points we argue as follows (see also Figure 4). Applying Lemma 7 we obtain that $\left.\partial_{1} W_{2}\right|_{\partial I_{E}^{1} \times I_{E}^{2}}>0$ and $\left.\partial_{2} W_{2}\right|_{I_{E}^{1} \times \partial I_{E}^{2}}>0$. In order to successfully apply Conley's Morse theory we need to choose an appropriate subset of $D_{E, 3}$ which will serve as an isolating neighborhood. Near $\left(u_{1}, u_{2}\right)=\left(u_{*}, u_{*}\right)$ we can find a small solution of the Euler-Lagrangian equation by perturbing from a linear solution. Consider the unique monotone lap $u(t)$ for which $u(0)=u_{1}=u_{*}-\delta$ and $u(\tau)=u_{*}+\delta$. Since $u_{*}$ is critical point of saddle-focus type, it follows that $u^{\prime \prime \prime}(0)<0$ and $u^{\prime \prime \prime}(\tau)<0$ for $\delta$ sufficiently small 27 . Straightforward calculation shows that $\partial_{1} \partial_{2} W_{2}=\partial_{1} \partial_{2} S\left(u_{1}, u_{2}\right)+\partial_{1} \partial_{2} S\left(u_{2}, u_{1}\right)>$ 0 . These two facts combined show that $\partial_{1} W_{2}\left(u_{*}-\delta, u_{2}\right)<0$ for all $u_{2} \leq u_{*}+\delta$, and $\partial_{2} W_{2}\left(u_{1}, u_{*}+\delta\right)>0$ for all $u_{1} \geq u_{*}-\delta$. Define $N_{\delta}=D_{E, 3}^{+} \backslash\left\{\left(u_{1}, u_{2}\right) \mid u_{*}-\delta<\right.$ $u_{1}\left\langle u_{*}, u_{*}+\delta>u_{2}>u_{*}\right\}$. The set $N_{\delta}$ is a closed subset of $D_{E, 3}^{+}$and is isolating with respect to the gradient flow of $W_{2} 28$ The next step is to compute the Conley index of the maximal invariant set $\operatorname{Inv}\left(N_{\delta}\right) \subset N_{\delta}$. It suffices here to compute the homological index (see [10]) of $\operatorname{Inv}\left(N_{\delta}\right)$. In order to do so we need to find an index pair for $\operatorname{Inv}\left(N_{\delta}\right)$. Let $\partial I_{E}^{1}=\left\{a_{1}^{-}, a_{1}^{+}\right\}, \partial I_{E}^{2}=\left\{a_{2}^{-}, a_{2}^{+}\right\}$. Let $N_{\delta}^{-}=\left\{u_{1}=u_{*}, u_{*}+\delta \leq u_{2} \leq a_{2}^{+}\right\} \cup\left\{a_{1}^{-} \leq u_{1} \leq u_{*}-\delta, u_{2}=u_{*}\right\}$, then $\left(N_{\delta}, N_{\delta}^{-}\right)$

\footnotetext{
${ }^{27}$ This follows for example from an explicit calculation of the solution for the linearized problem.

${ }^{28}$ The flow is not well-defined on the boundary of $D_{E, 3}$, but we can choose a slightly smaller isolating neighborhood inside $D_{E, 3}$ with the same Conley index (alternatively we can use the Morse index for $C^{1}$-functions (see also Section 2.3) ).
} 
is an index pair for $\operatorname{Inv}\left(N_{\delta}\right)$, and $C H_{*}\left(\operatorname{Inv}\left(N_{\delta}\right)\right)=H_{*}\left(N_{\delta}, N_{\delta}^{-}\right)$. Consequently $C H_{1}\left(\operatorname{Inv}\left(N_{\delta}\right)\right) \simeq \mathbb{Z}$ and $C H_{k}\left(\operatorname{Inv}\left(N_{\delta}\right)\right)=0$ for $k \neq 1$. The fact that the homological Conley index is non-trivial for $k=1$ and because (8) is a gradient flow we conclude that there exists at least one critical point of $W_{2}$ in $N_{\delta}$ with large Morse index equal to 1 .

With regard to the relative position of the extrema of $W_{2}$ we note the following. Let $\left(b_{i}, c_{i}\right)$ be the maximum in $D_{E, i}^{+}$for $i=1,2$. Since $\nabla W_{2}\left(b_{i}, c_{i}\right)=0$ it follows from Lemma 5 part (2) that $\partial_{1} W_{2}\left(b_{1}, u_{2}\right)>0$ for all $u_{2}>c_{1}$ and $\partial_{2} W_{2}\left(u_{1}, c_{2}\right)<0$ for all $u_{1}<b_{2}$. Therefore, we may as well use $\tilde{D}_{E, 3}^{+}=\left\{b_{1} \leq u_{1} \leq u_{*}, u_{*} \leq u_{2} \leq\right.$ $\left.c_{2}\right\} \backslash\left(u_{*}, u_{*}\right)$ instead of $D_{E, 3}^{+}$. We then obtain a saddle point $\left(b_{3}, c_{3}\right) \in \tilde{D}_{E, 3}^{+}$with $b_{1}<b_{3}<b_{2}$ and $c_{1}<c_{3}<c_{2}$.

In terms of closed characteristics for a Lagrangian system the above lemma yields

Theorem 16. Let $\pi^{u} N_{E}$ contain a concatenation $I_{E}^{\#}$ of two compact intervals $I_{E}^{1}$ and $I_{E}^{2}$, and assume that $(L, d t)$ is a Twist system on $I_{E}^{\#}$. If $u_{*} \in I_{E}^{1} \cap I_{E}^{2}$ is of saddle-focus type, then there exist at least 3 geometrically distinct closed characteristics.

An analogue of the above theorem can also be proved for concatenations of more than two interval components. We leave this to the interested reader.

3.4. Non-compact interval components. As already indicated in the previous sections the theory developed in this paper is applicable to various model equations that we know from physics, such as the eFK/Swift-Hohenberg type equations, 5th order KdV equations, suspension bridge model, etc. (see Section 2.2). In this section we will take a closer look at the class of eFK/Swift-Hohenberg type equations. This family of equations is given by a Lagrangian of the form: $L\left(u, u^{\prime}, u^{\prime \prime}\right)=$ $\frac{1}{2}\left|u^{\prime \prime}\right|^{2}+\frac{\alpha}{2}\left|u^{\prime}\right|^{2}+F(u)$, where $F$ is the potential, which is an arbitrary $C^{2}$-function of $u$. We have already proved that such Lagrangian systems are always Twist systems if $\alpha \leq 0$ (and we believe the same to be true also for $\alpha>0$ (Twist property on interval components)). The results obtained in this paper prove that for any energy level $E$ for which the set $\{u \mid F(u)+E \geq 0\}$ contains a compact interval component $I_{E}$, there exist a simple closed characteristic $u(t) \in \operatorname{int}\left(I_{E}\right)$. Let us by means of example consider a double equal-well potential $F\left(\right.$ like $\left.\frac{1}{4}\left(u^{2}-1\right)^{2}\right)$ with $\min _{u} F(u)=0$. In this case the set $\{u \mid F(u)+E \geq 0\}$ always contains noncompact interval components. Without further geometric knowledge of the energy manifold $M_{E}$ a general topological result proving existence of closed characteristics does not seem likely. Therefore we will consider a specific example here. Consider the energy level $E=0$, then $I_{0} \stackrel{\text { def }}{=} \pi^{u} N_{0}=\mathbb{R}$, and $I_{0}$ is a concatenation of three interval components. The Lagrangian system with $\alpha \leq 0$ is a Twist system on $I_{0}$ and therefore $S$ is well-defined on $\mathbb{R}^{2}$. One way to deal with this non-compact case is to compactify the system (see [14]). This however requires detailed information about the asymptotic behavior of $F$. There is a weaker assumption that one can use in order to restrict the analysis of $W_{2}$ to a compact subset of $D_{E}^{+}$. This boils down to the following geometric property:

(D) There exists a pair $\left(u_{1}^{*}, u_{2}^{*}\right) \in D_{E}^{+}$(with $\left|u_{1}^{*}\right|$ and $\left|u_{2}^{*}\right|$ large) such that $u_{a, b}^{\prime \prime \prime}(0)<$ 0 and $u_{a, b}^{\prime \prime \prime}(\tau)<0$ for the unique minimizers $u_{a}=u\left(t ; u_{1}^{*}, u_{2}^{*}\right)$ and $u_{b}=$ $u\left(t ; u_{2}^{*}, u_{1}^{*}\right)$ of $[6)^{29}$.

\footnotetext{
${ }^{29}$ Notice that $u_{a}(t)=u_{2}^{*}-u_{b}(\tau-t)$ if $L(u, v, w)$ is symmetric in $v$.
} 


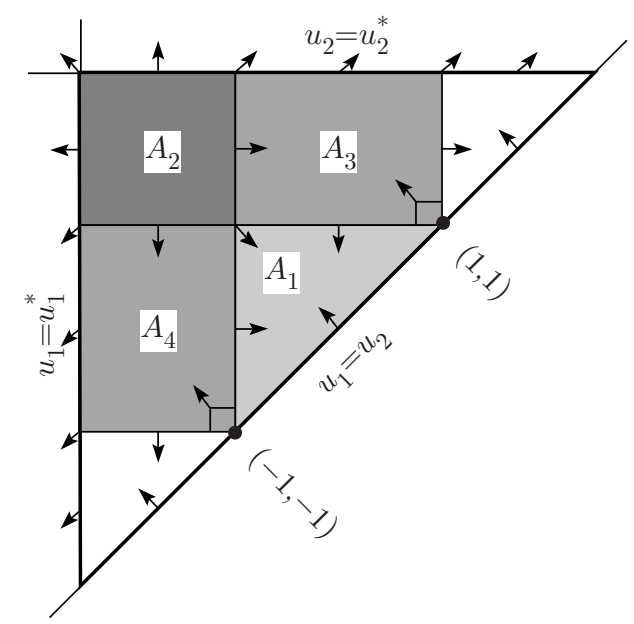

Figure 5. The triangle $D_{E}^{+}=I_{E}^{*} \times I_{E}^{*} \cap\left\{u_{2}>u_{1}\right\}$ for the case of a double-well potential. The arrows denote (schematically) the direction of the gradient $\nabla W_{2}$. Clearly $W_{2}$ has at least one maximum and one minimum. Additionally, when the equilibrium points are saddle-foci then $W_{2}$ has two saddle points.

If $(L, d t)$ satisfies Hypothesis (D) on a (non-compact) interval component $I_{E}$, then the system is said to be dissipative on $I_{E}^{*}=\left[u_{1}^{*}, u_{2}^{*}\right]$.

Lemma 17. If a Lagrangian system is dissipative on $I_{E}^{*}$, then it holds that $\partial_{1} W_{2}\left(u_{1}^{*}, u_{2}\right)<0$ for all $u_{2} \in\left(u_{1}^{*}, u_{2}^{*}\right]$ and $\partial_{2} W_{2}\left(u_{1}, u_{2}^{*}\right)>0$ for all $u_{1} \in\left[u_{1}^{*}, u_{2}^{*}\right)$.

Proof. It follows from (D) that $\partial_{1} W_{2}\left(u_{1}^{*}, u_{2}^{*}\right)<0$. Lemma 5 part (2) implies that $\partial_{1} W_{2}\left(u_{1}^{*}, u_{2}\right)$ is increasing as a function of $u_{2}$. It easily follows that $\partial_{1} W_{2}\left(u_{1}^{*}, u_{2}\right)<$ 0 for all $u_{2} \leq u_{2}^{*}$. The other assertion is proved in exactly the same way.

For many nonlinearities $F(u)$ it can be proved that the eFK/Swift-Hohenberg system is dissipative on some interval $I_{E}^{*}=\left[u_{1}^{*}, u_{2}^{*}\right]$ with $u_{1}^{*}<-1$ and $u_{2}^{*}>$ +1 30. Notice that $S$ need not have any critical points, for example for $E \gg 0$ (see [14]). For $E=0$ there are two equilibrium points which will force $S$ to have critical points.

Lemma 18. If the Swift-Hohenberg Lagrangian is dissipative on $I_{0}^{*}$ (with $\{ \pm 1\}$ $\left.\in I_{0}^{*}\right)$, then it has at least two geometrically distinct simple closed characteristics (large and small amplitude). Moreover, if $u= \pm 1$ are both saddle-foci then there exist two more geometrically distinct simple closed characteristics.

Proof. We consider the function $W_{2}$ on $I_{E}^{*} \times I_{E}^{*}$ and as before we define $D_{E}^{+}=$ $I_{E}^{*} \times I_{E}^{*} \cap\left\{u_{2}>u_{1}\right\}$ (see also Figure 5). Define $A_{1}=\left\{-1<u_{1}<u_{2}<1\right\}$ and $A_{2}=D_{E}^{+} \cap\left\{u_{1}<-1, u_{2}>1\right\}$. As in the proof of Lemma 11 we have that $\partial_{1} W_{2}\left( \pm 1, u_{2}\right)>0$ and $\partial_{2} W_{2}\left(u_{1}, \pm 1\right)<0$. We now see from Lemma 17 that the

\footnotetext{
${ }^{30}$ For example, when $F(u) \sim|u|^{n}$ as $|u| \rightarrow \infty$ for some $n>2$ then this follows from a scaling argument. After scaling the Euler-Lagrange equation tends to $u^{\prime \prime \prime \prime}=-|u| u^{n-2}$. For this equation it is easy to see that $u(0)=u_{1}<0, u^{\prime \prime \prime}(0)=0$ implies that $u(\tau)=u_{2}>0$ and $u^{\prime \prime \prime}(\tau)<0$. A perturbation argument then shows that (D) is satisfied for the original equation for some $\left(u_{1}^{*}, u_{2}^{*}\right)$ with $-u_{1}^{*}$ and $u_{2}^{*}$ large.
} 
gradient of $W_{2}$ points outwards on $\partial A_{2}$ and inwards on $\partial A_{1}$. Hence, on $A_{1}$ the function $W_{2}$ attains a maximum and on $A_{2}$ the function $W_{2}$ attains a minimum (index 2 and index 0 points respectively), which proves the first part of the lemma.

As for the second part we argue as in the proof of Lemma 13. Since $u= \pm 1$ are saddle-foci one finds index 1 saddle points in both $A_{3}=D_{E}^{+} \cap\left\{-1<u_{1}<1, u_{2}>1\right\}$ and $A_{4}=D_{E}^{+} \cap\left\{u_{1}<-1,-1<u_{2}<1\right\}$.

Concerning the relative position of the extrema of $W_{2}$, the same reasoning as at the end of Section 3.3 can be followed. Denoting by $\left(b_{i}, c_{i}\right)$ the extremum in $A_{i}$ (for $i=1,2,3,4)$ we find that $b_{2}<b_{4}<b_{1}<b_{3}$ and $c_{4}<c_{1}<c_{3}<c_{2}$.

The result proved above has already been found in [24, 31] for the special case $F(u)=\frac{1}{4}\left(u^{2}-1\right)^{2}$ without information about the index of the solutions. Many more examples can be considered with non-compact interval components. A rather tricky system is the suspension bridge model (see Section 2.2.2). The Lagrangian is given by $L\left(u, u^{\prime}, u^{\prime \prime}\right)=\frac{1}{2}\left|u^{\prime \prime}\right|^{2}-\frac{c^{2}}{2}\left|u^{\prime}\right|^{2}+F(u)$, where $F(u)=e^{u}-u-1$. This nonlinearity is especially hard to deal with when trying to compactify $D_{E}^{+}$. In this context it is interesting to note that there is no a priori $L^{\infty}$ bound on the set of bounded solutions (see 30]) as opposed to nonlinearities with super-quadratic growth. From the analysis in [30] it follows that there exists a point $\left(u_{1}^{*}, u_{2}^{*}\right) \in D_{E}^{+}$ such that $\partial_{1} S\left(u_{1}^{*}, u_{2}^{*}\right)>0, \partial_{2} S\left(u_{1}^{*}, u_{2}^{*}\right)>0$, and $\partial_{1} S\left(u_{1}^{*}, u_{2}\right)>0$ for all $u_{2}>0$. This is a different dissipativity condition. Upon examining $W_{2}$ (for $E=0$ ) on $I_{E}^{*} \times I_{E}^{*}$ we find at least one index 1 simple closed characteristic for the suspension bridge problem (this was already proved in [30, without information on the Morse index). In order for the argument to work the equilibrium point 0 has to be a saddle-focus. Moreover, for the dissipativity condition to be satisfied the coefficient in front of the second term in the Lagrangian has to be strictly positive. In [30] more complicated closed characteristics are also found. This will be the subject of further study.

\section{Concluding Remarks}

4.1. Numerical evidence for the Twist property. In Lemma 9 we prove the Twist property for a class of Lagrangians including the well-known Swift-Hohenberg Lagrangian. Numerical evidence suggests that the Twist property holds for a large class of other Lagrangians as well. As an example we depict in Figure 6] solutions of the eFK equation (i.e., the eFK Lagrangian with $\left.F(u)=\frac{1}{4}\left(u^{2}-1\right)^{2}\right)$. For $\alpha \leq 0$ the Twist property is always satisfied by Lemma 9 Numerical evidence suggests that the Twist property is satisfied for all $E>0$ and all $\alpha \in \mathbb{R}$ (with $I_{E}=\mathbb{R}$ ). At the singular energy level $E=0$ there are (for $\alpha>0$ ) two different cases, namely where the equilibrium points are real saddles and saddle-foci. While the Twist property certainly is not satisfied on the whole of $\mathbb{R}$ (it $i s$ satisfied on the interval component $[-1,1])$ for the real saddle case, we conjecture that the Twist property holds on $\mathbb{R}$ as long as the equilibrium points are saddle-foci.

We also performed numerical calculations on the 5 th order KdV equation (see Section 2.2.3) and it seems that the same is true for this system. It is of course impossible to make statements about the rich class of second order Lagrangians as a whole, but the Twist property appears to hold for a large subclass.

4.2. Local behavior at equilibrium points. In Section 3.2 we indicated that the critical points $u_{*}$ with $\partial_{u}^{2} L\left(u_{*}, 0,0\right)>0$ can be categorized into three classes: 

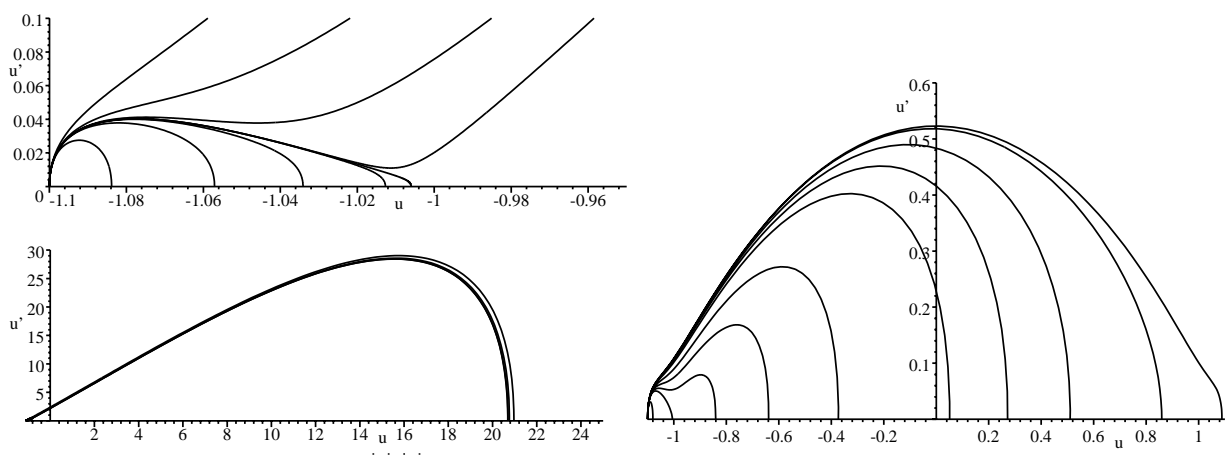

Figure 6. For fixed $u_{1}=-1.1$ characteristics in the energy level $E=0$ of the eFK Lagrangian are shown (in the $\left(u, u^{\prime}\right)$-plane). On the left the equilibrium points $u= \pm 1$ are real saddles $(\alpha=5)$. Notice the different scales needed to obtain an overall picture of the situation. The Twist property is only satisfied for $u_{2} \in\left(u_{1},-1\right)$. On the right the equilibrium points are saddle-foci $(\alpha=1)$. In this case the Twist property seemingly holds for all $u_{2}>u_{1}$.

$\sigma\left(u_{*}\right)=\left\{ \pm \lambda_{1}, \pm \lambda_{2}\right\}$ (real saddle), $\sigma\left(u_{*}\right)=\{ \pm a \pm b i\}$ (saddle-focus), and $\sigma\left(u_{*}\right)=$ $\{ \pm a i, \pm b i\}$ (center). The fourth possibility, which occurs for equilibrium points with $\partial_{u}^{2} L\left(u_{*}, 0,0\right)<0$, is $\sigma\left(u_{*}\right)=\{ \pm \lambda, \pm a i\}$ (saddle-center). Such points do however not occur as boundary points of interval components and one may ask how they fit in.

Consider a compact interval component $I_{E}$, then $L(u, 0,0)+E>0$ for all $u \in$ $\operatorname{int}\left(I_{E}\right)$ and $\left.\partial_{u} L\right|_{\partial I_{E}} \geq 0$ (if $\partial_{u} L=0$ at a boundary point then necessarily $\partial_{u}^{2} L>0$ ). There exists a point $u_{*} \in \operatorname{int}\left(I_{E}\right)$ such that $\partial_{u} L\left(u_{*}, 0,0\right)=0$ and $\partial_{u}^{2} L\left(u_{*}, 0,0\right)<0$. As a matter of fact there may be many minima and maxima. Now let $E$ decrease until the next singular level is reached. If the extremum in this level is a minimum then $I_{E}$ splits into two components, and if this extremum is a maximum then $I_{E}$ simply shrinks to the point $u_{*}$. Conversely, if $u_{*}$ is a saddle-center equilibrium point at energy level $E_{*}$, then there exists an $\epsilon>0$ such that $\pi^{u} N_{E_{*}+\epsilon}$ contains a compact interval component $I_{E_{*}+\epsilon}$ which shrinks to $u_{*}$ as $\epsilon \rightarrow 0$.

The local theory for saddle-centers reveals the existence of a family of closed characteristics on $I_{E_{*}+\epsilon}$ parametrized by $\epsilon$ (Lyapunov Center Theorem). Our theory not only provides the existence of closed characteristics for $E_{*}<E<E_{*}+\epsilon$ but also guarantees the existence of closed characteristics for all $E>E_{*}$ as long as the interval component $I_{E}$ containing $u_{*}$ remains compact. We should emphasize again the resemblance with the classical mechanical system $\frac{\partial L\left(u, u^{\prime}\right)}{\partial u}-\frac{d}{d t} \frac{\partial L\left(u, u^{\prime}\right)}{\partial u^{\prime}}=0$.

4.3. KAM theory. For the Lagrangian systems that we study in this paper one may wonder whether such systems can be completely integrable. A Lagrangian system $(L, d t)$ is said to be completely integrable if the associated Hamiltonian system $(H, \omega)$ is completely integrable. Many of the examples that we consider such as the eFK/Swift-Hohenberg system with $\alpha \leq 0$ are far from being integrable. An example of an integrable system is given by the Lagrangian $L\left(u, u^{\prime}, u^{\prime \prime}\right)=$ $\frac{1}{2}\left|u^{\prime \prime}\right|^{2}+\frac{1}{4} u^{4}$ (see [14] for a proof). Integrability can also be addressed at the 
level of the Twist maps in the Lagrangian systems. Without going into too much detail let us look at a specific example. Consider again the eFK/Swift-Hohenberg family defined by the $L\left(u, u^{\prime}, u^{\prime \prime}\right)=\frac{1}{2}\left|u^{\prime \prime}\right|^{2}+\frac{\alpha}{2}\left|u^{\prime}\right|^{2}+\frac{1}{4}\left(u^{2}-1\right)^{2}, \alpha \leq 0$. Now let $E<0$ and consider the area-preserving map $T$ on $\mathbb{R}^{2}$ as discussed in Section 3.3. It follows from the compactification results in 14 that $\mathbb{R}^{2} \backslash B_{r}(0)$ contains only invariant curves for the map $T$ for $r>0$ sufficiently large. Inside the ball $B_{r}(0)$ the map $T$ can be chaotic (depending on the nature of the equilibrium points). The invariant curves in $\mathbb{R}^{2} \backslash B_{r}(0)$ can be interpreted as the invariant tori/circles of an integrable system, comparable to the conserved invariant tori in KAM theory. To get a feel for integrability of the map $T$ on compact interval components we can look at the quadratic Lagrangian $L\left(u, u^{\prime}, u^{\prime \prime}\right)=\frac{1}{2}\left|u^{\prime \prime}\right|^{2}-\frac{1}{2} u^{2}$. We will leave this to the interested reader.

The question of integrability versus non-integrability for second order Lagrangian systems may be fairly complex. The results in [17, 18] and those proved in Section 3.3 seem to suggest that equilibrium points of saddle-focus and center type in combination with geometric and topological conditions on the system create regions of non-integrability. With the techniques presented in this paper and the methods in [12, 13] we are trying to understand some of the dynamics of the system in this case. These questions will be subject of future study.

\section{Appendix A. Classification of EQUilibrium Points}

The equilibrium solutions of the Euler-Lagrange equation,

$$
\frac{\partial L}{\partial u}-\frac{d}{d t} \frac{\partial L}{\partial u^{\prime}}+\frac{d^{2}}{d t^{2}} \frac{\partial L}{\partial u^{\prime \prime}}=0
$$

are given by the relation $\frac{\partial L}{\partial u}\left(u_{*}, 0,0\right)=0$. The sign of $\frac{\partial^{2} L}{\partial u^{2}}\left(u_{*}, 0,0\right)$ divides the behaviors of the equilibrium points into two groups. We will not consider the case $\frac{\partial^{2} L}{\partial u^{2}}\left(u_{*}, 0,0\right)=0$ which requires information on higher order derivatives. Equilibrium points for which $\frac{\partial^{2} L}{\partial u^{2}}\left(u_{*}, 0,0\right) \neq 0$ are usually called non-degenerate. In order to study the local structure of singular points we need to consider the second variation of $J[u]$ around an equilibrium solution $u(t) \equiv u_{*}$. This yields the following linear differential equation for the variations $\varphi$ :

$$
\frac{\partial^{2} L}{\partial u^{2}} \varphi+\left(2 \frac{\partial^{2} L}{\partial u \partial u^{\prime \prime}}-\frac{\partial^{2} L}{\partial{u^{\prime}}^{2}}\right) \varphi^{\prime \prime}+\frac{\partial^{2} L}{\partial{u^{\prime \prime}}^{2}} \varphi^{\prime \prime \prime \prime}=0,
$$

where all partial derivatives of $L$ are evaluated at $\left(u, u^{\prime}, u^{\prime \prime}\right)=\left(u_{*}, 0,0\right)$. The characteristic equation is given by $\partial_{u}^{2} L+\left(2 \partial_{u u^{\prime \prime}}^{2} L-\partial_{u^{\prime}}^{2} L\right) \lambda^{2}+\left(\partial_{u^{\prime \prime}}^{2} L\right) \lambda^{4}=0$. For non-degenerate equilibrium solutions the following classification holds:

Lemma 19. Let $u(t) \equiv u_{*}$ be an equilibrium solution.

(i) If $\partial_{u}^{2} L<0$, then $\sigma\left(u_{*}\right)=\{ \pm \lambda, \pm$ ai $\}$ (saddle-center).

(ii) If $\partial_{u}^{2} L>0$, then $\sigma\left(u_{*}\right)=\left\{ \pm \lambda_{1}, \pm \lambda_{2}\right\}, \sigma\left(u_{*}\right)=\{ \pm a i, \pm b i\}$, or $\sigma\left(u_{*}\right)=$ $\{ \pm a \pm b i\}$ (real saddle, center, and saddle-focus respectively) depending on $\partial_{u u^{\prime \prime}}^{2} L$ and $\partial_{u^{\prime}}^{2} L$.

Here $a, b, \lambda, \lambda_{1}, \lambda_{2}>0$.

Proof. From the characteristic equation we derive

$$
\lambda_{ \pm}^{2}=\frac{-\left(2 \partial_{u u^{\prime \prime}}^{2} L-\partial_{u^{\prime}}^{2} L\right) \pm \sqrt{D}}{2 \partial_{u^{\prime \prime}}^{2} L},
$$


where $D=\left(2 \partial_{u u^{\prime \prime}}^{2} L-\partial_{u^{\prime}}^{2} L\right)^{2}-4\left(\partial_{u^{\prime \prime}}^{2} L\right)\left(\partial_{u}^{2} L\right)$. Clearly if $\partial_{u}^{2} L<0$, then $\sqrt{D}>$ $\left|2 \partial_{u u^{\prime \prime}}^{2} L-\partial_{u^{\prime}}^{2} L\right|$ and thus $\lambda_{-}^{2}<0$ and $\lambda_{+}^{2}>0$. This forces the spectrum to be $\{ \pm \lambda, \pm a i\}$. If $\partial_{u}^{2} L>0$, then $\sqrt{|D|}<\left|2 \partial_{u u^{\prime \prime}}^{2} L-\partial_{u^{\prime}}^{2} L\right|$ and there are 3 possibilities:

1. $D>0$, then $\sqrt{D}<\left|2 \partial_{u u^{\prime \prime}}^{2} L-\partial_{u^{\prime}}^{2} L\right|$ and $\lambda_{ \pm}^{2}$ are both positive or negative. This depends on $\partial_{u u^{\prime \prime}}^{2} L$ and $\partial_{u^{\prime}}^{2} L$. If both eigenvalues are negative the spectrum is given by $\{ \pm a i, \pm b i\}$, and if both eigenvalues are positive the spectrum is $\left\{ \pm \lambda_{1}, \pm \lambda_{2}\right\}$.

2. $D=0$, then the same possibilities as in the previous case hold, with the additional property that the eigenvalues all have multiplicity two.

3. $D<0$, then $\lambda_{ \pm}^{2} \in \mathbb{C} \backslash \mathbb{R}$ and therefore the spectrum is $\{ \pm a \pm b i\}$.

As indicated before we do not study the case $\partial_{u}^{2} L=0$. In order to analyze degenerate equilibrium solutions a normal form analysis is required. An example of such type of analysis for a non-linear saddle-focus can be found in [14]. The results proved in 14 for non-linear saddle-foci would suffice for the purposes of this paper.

\section{Appendix B. The proof of Lemma 9}

Stationary functions of the action functional $J_{E}[u]$, with $L\left(u, u^{\prime}, u^{\prime \prime}\right)=\frac{1}{2}\left|u^{\prime \prime}\right|^{2}+$ $K\left(u, u^{\prime}\right)$, satisfy the equation

$$
u^{\prime \prime \prime \prime}-\frac{d}{d t} \frac{\partial K}{\partial u^{\prime}}+\frac{\partial K}{\partial u}=0 .
$$

Solutions of (11) satisfy the Hamiltonian relation $-u^{\prime} u^{\prime \prime \prime}+\frac{1}{2}\left|u^{\prime \prime}\right|^{2}+\frac{\partial K}{\partial u^{\prime}} u^{\prime}-K\left(u, u^{\prime}\right)-$ $E=0$. For an increasing lap from $u_{1}$ to $u_{2}$ the derivative $u^{\prime}$ can be represented as a function of $u$. Set $z(u)=u^{\prime} \sqrt{u^{\prime}}$ (see for example [5, 28] where similar transformations are used). Using the Hamiltonian relation we find that $z$ satisfies the equation $z^{\prime \prime}=g(u, z), z>0, z\left(u_{1}\right)=z\left(u_{2}\right)=0$, where $g(u, z)=\frac{3}{2} \frac{\frac{\partial K}{\partial u^{\prime}} u^{\prime}-K\left(u, u^{\prime}\right)-E}{z^{5 / 3}}$. The same holds for decreasing laps $(z<0)$. If

$$
\frac{\partial K}{\partial u^{\prime}} u^{\prime}-K\left(u, u^{\prime}\right)-E \leq 0, \quad \text { and } \quad \frac{\partial^{2} K}{\partial{u^{\prime}}^{2}} u^{\prime 2}-\frac{5}{2}\left(\frac{\partial K}{\partial u^{\prime}} u^{\prime}-K\left(u, u^{\prime}\right)-E\right) \geq 0,
$$

for all $u \in I_{E}$, and $z \geq 0$ (condition (a) and (b) in Lemma 9), then $g(u, z) \leq 0$, and $\frac{\partial g}{\partial z}(u, z) \geq 0$ respectively.

It follows from results in [11] that the boundary value problem for the $z$-equation has a unique strictly concave positive solution. Consequently the $u$-laps from $u_{1}$ to $u_{2}$ are unique, and we thus obtain a family $u\left(t ; u_{1}, u_{2}\right)$. These functions are global minimizers of $J_{E} 31$. From the smooth dependence of the initial value problem of (11) we deduce that these functions depend continuously on $\lambda=\left(u_{1}, u_{2}\right) \in \Lambda \stackrel{\text { def }}{=}$ $I_{E} \times I_{E} \backslash \Delta$, and that the time $\tau\left(u_{1}, u_{2}\right)$ it takes for $u$ to (monotonically) go from $u_{1}$ to $u_{2}$ depends continuously on $u_{1}$ and $u_{2}$ as well 32 and $\tau\left(u_{1}, u_{2}\right)<\infty$ for all $\left(u_{1}, u_{2}\right) \in \Lambda^{33}$.

The remainder of this proof will be concerned with showing that $u(t ; \lambda)$ varies smoothly with respect to $\lambda$ for all $\lambda \in \operatorname{int}(\Lambda)$ that are away from possible equilibrium

\footnotetext{
${ }^{31}$ In $z$-variables we have $J_{E}=\int_{u_{1}}^{u_{2}}\left(\frac{2}{9} z^{\prime 2}+\frac{K\left(u, z^{2 / 3}\right)+E}{z^{2 / 3}}\right) d u$. The condition $\frac{\partial g}{\partial z} \geq 0$ implies that this functional is convex.

${ }^{32}$ Away from equilibrium points this is obvious. At equilibrium points this follows either by taking limits and using the uniqueness, or from the local analysis performed in [29] Lemma 5.8].

${ }^{33}$ It follows from $g \leq 0$ and the analysis in Appendix $\mathrm{A}$ that equilibrium points (which are non-degenerate by assumption) can only be of saddle-focus or center type.
} 
points. Rescale the $u$-variable as $s=\frac{u-u_{1}}{u_{2}-u_{1}}$ and set $y(s)=z(u)$. From the $z$ equation we obtain the following equation for $y$ :

$$
y^{\prime \prime}=\tilde{g}(s, y ; \lambda), y(0)=y(1)=0, y>0 \text { on }(0,1) .
$$

Moreover $\tilde{g} \leq 0$ and $\frac{\partial \tilde{g}}{\partial y} \geq 0$, and we can write $\tilde{g}(s, u ; \lambda)=\frac{h(s, y ; \lambda)}{y^{5 / 3}}$ with $h(s, y ; \lambda)$ a continuous function.

In order to obtain smooth dependence on the parameter $\lambda$ we first consider the following equation: $y_{\epsilon}^{\prime \prime}=\tilde{g}\left(s, y_{\epsilon} ; \lambda\right), y_{\epsilon}(0)=y_{\epsilon}(1)=\epsilon$ and $y_{\epsilon}>\epsilon$ on $(0,1)$. It follows from the maximum principle that $0<y_{\epsilon}-y_{0} \leq \epsilon$. For the $y_{\epsilon}$-problem it is not difficult to show that $y_{\epsilon}(\cdot ; \lambda)$ depends smoothly on $\lambda$. To prove this we consider the map $F\left(y_{\epsilon}, \lambda\right)=y_{\epsilon}^{\prime \prime}-g\left(s, y_{\epsilon} ; \lambda\right)$, where $F$ maps from $X_{\epsilon} \times \Lambda$ (with $\left.X_{\epsilon}=\epsilon+H_{0}^{1}(0,1)\right)$ to $H^{-1}(0,1)$, and $F \in C^{1}\left(X_{\epsilon} \times \Lambda, H^{-1}\right)$. From the Implicit Function Theorem we derive that

$$
\frac{d}{d \lambda} y_{\epsilon}(\cdot ; \lambda)=-\left(F_{y}\left(y_{\epsilon}, \lambda\right)\right)^{-1} F_{\lambda}\left(y_{\epsilon}, \lambda\right) \in C\left(\Lambda, X_{\epsilon}\right)
$$

Our goal now is to derive a similar expression for $\frac{d}{d \lambda} y_{0}(\cdot ; \lambda)$. We cannot apply the Implicit Function Theorem to $y_{0}$ directly because of the singularity of $\tilde{g}$ at $y=0$.

We define $\Phi_{\epsilon}(\lambda) \stackrel{\text { def }}{=} F_{y}\left(y_{\epsilon}(\cdot ; \lambda), \lambda\right)=\frac{d^{2}}{d s^{2}}-\frac{\partial g}{\partial y}\left(s, y_{\epsilon} ; \lambda\right)=\frac{d^{2}}{d s^{2}}-\frac{k\left(s, y_{\epsilon} ; \lambda\right)}{y_{\epsilon}^{8 / 3}}$, where $k$ is a continuous function. For $\lambda \in \Lambda$ away from the equilibrium points the asymptotic behavior of $y_{0}$ at $s=0,1$ is $y_{0}(s)=O\left(s^{3 / 4}\right)$ as $s \downarrow 0$ and $y_{0}(s)=O\left((1-s)^{3 / 4}\right)$ as $s \uparrow 1$. We now conclude from Hardy's inequality that $\Phi_{0}(\lambda) \in B\left(H_{0}^{1}, H^{-1}\right)$ for all $\lambda \in \Lambda$.

It holds that $\Phi_{\epsilon}(\lambda) \longrightarrow \Phi_{0}(\lambda)$ in $B\left(H_{0}^{1}, H^{-1}\right)$ as $\epsilon \rightarrow 0$, and the same holds for the inverses in $B\left(H^{-1}, H_{0}^{1}\right)$ since $\Phi_{\epsilon}(\lambda)$ is uniformly bounded in $\epsilon$. We obtain that (writing $k_{\epsilon}=k\left(\cdot, y_{\epsilon} ; \lambda\right)$ )

$$
\left\|\Phi_{\epsilon}(\lambda)-\Phi_{0}(\lambda)\right\| \leq C\left\|k_{\epsilon}\left(\frac{y_{0}}{y_{\epsilon}}\right)^{\frac{8}{3}}-k_{0}\right\|_{L^{2}} .
$$

From the $L^{\infty}$-convergence of $y_{\epsilon}$ to $y_{0}$ we then conclude that $\Phi_{\epsilon}(\lambda) \rightarrow \Phi_{0}(\lambda)$ as $\epsilon \rightarrow 0$. In order to obtain the above inequality we again used Hardy's inequality in combination with the asymptotic behavior of $y_{0}$ at $s=0,134$.

We now assert that $F_{\lambda}\left(y_{\epsilon}, \lambda\right) \longrightarrow F_{\lambda}\left(y_{0}, \lambda\right)$ in $H^{-1}$ as $\epsilon \rightarrow 0$. We find that

$$
\left\|F_{\lambda}\left(y_{\epsilon}, \lambda\right)-F_{\lambda}\left(y_{0}, \lambda\right)\right\|_{H^{-1}} \leq C\left\|\frac{\partial h_{\epsilon}}{\partial \lambda}\left(\frac{y_{0}}{y_{\epsilon}}\right)^{\frac{5}{3}}-\frac{\partial h_{0}}{\partial \lambda}\right\|_{L^{2}} .
$$

As before, due to the $L^{\infty}$-convergence of $y_{\epsilon}$ to $y_{0}$ the assertion follows.

We conclude that $\frac{d}{d \lambda} y_{\epsilon}(\cdot ; \lambda)$ converges to $\left(F_{y}\left(y_{0}, \lambda\right)\right)^{-1} F_{\lambda}\left(y_{0}, \lambda\right) \stackrel{\text { def }}{=} \zeta_{\lambda}$. The next step is to consider the difference quotient $D_{h} y(\cdot ; \lambda)=\frac{y(\cdot ; \lambda+h)-y(\cdot ; \lambda)}{h}$. We have that $D_{h} y_{\epsilon} \rightarrow D_{h} y_{0}$ in $L^{\infty}$ as $\epsilon \rightarrow 0$, and $D_{h} y_{\epsilon} \rightarrow \frac{d}{d \lambda} y_{\epsilon}(\cdot ; \lambda)$ as $h \rightarrow 0$ for $\epsilon>0$. Combining these facts we obtain $\left\|\zeta_{\lambda}-D_{h} y_{0}\right\|_{L^{\infty}} \leq\left\|\zeta_{\lambda}-\frac{d}{d \lambda} y_{\epsilon}(\cdot ; \lambda)\right\|_{L^{\infty}}+$ $\left\|\frac{d}{d \lambda} y_{\epsilon}(\cdot ; \lambda)-D_{h} y_{\epsilon}\right\|_{L^{\infty}}+\left\|D_{h} y_{\epsilon}-D_{h} y_{0}\right\|_{L^{\infty}} \rightarrow 0$ as $\epsilon, h \rightarrow 0$. This gives

$$
\frac{d}{d \lambda} y_{0}(\cdot ; \lambda)=-\left(F_{y}\left(y_{0}, \lambda\right)\right)^{-1} F_{\lambda}\left(y_{0}, \lambda\right) \in H_{0}^{1}(0,1), \forall \lambda \in \operatorname{int}(\Lambda) .
$$

Finally, an estimate similar to the ones above shows that $\frac{d}{d \lambda} y_{0}(\cdot ; \lambda)$ depends continuously on $\lambda$ for all $\lambda \in \operatorname{int}(\Lambda)$ that are away from equilibrium points. It then

\footnotetext{
${ }^{34}$ If $k$ has a zero at $s=0$ or $s=1$ the asymptotic behavior of $y_{0}$ will be different (i.e. $y_{0}=O(s)$ near $s=0)$. In this case a slightly different inequality holds which proves the same statement.
} 
follows from the differential equation that $y_{0}^{\prime}(s ; \lambda)$ and $y_{0}^{\prime \prime}(s ; \lambda)$ are $C^{1}$-functions of $\lambda$ for all $s \in(0,1)$, i.e., $y_{0}(\cdot ; \lambda)$ is continuously differentiable as a $C^{2}$-function on any compact subset of $(0,1)$. This implies that $u(\cdot ; \lambda)$ is continuously differentiable as a $C^{3}$-function (at least away from its extrema). Finally, a simple application of the Implicit Function Theorem shows that $\tau(\lambda)$ is continuously differentiable for all $\lambda \in \operatorname{int}(\Lambda)$ that are away from equilibrium points.

\section{REFERENCES}

[1] N.N. Akmediev, A.V. Buryak and M. Karlsson, Radiationless optical solitons with oscillating tails, Opt. Comm. 110 (1994), 540-544.

[2] S.B. Angenent, The periodic orbits of an area preserving Twist-map, Comm. Math. Phys. 115 (1988), 353-374. MR 89f:58118

[3] S.B. Angenent, Monotone recurrence relations, their Birkhoff orbits and topological entropy, Erg. Th. \& Dyn. Syst. 10 (1990), 15-41. MR 91b:58181

[4] V.I. Arnol'd and S.P. Novikov, Dynamical Systems IV, Springer Verlag 1990. MR 90j:58039

[5] V.I. Arnol'd, Mathematical methods of classical mechanics, Springer-Verlag, 1978. MR 96c:70001

[6] S. Aubry and P.Y. LeDaeron, The discrete Frenkel-Kontorova model and its extensions, Physica D 8 (1983), 381-422. MR 85f:58032

[7] V. Benci, A new approach to the Morse-Conley theory and some applications, Ann. Mat. Pura Appl. 158 (1991) 231-305. MR 92k:58043

[8] V. Benci and F. Giannoni Morse theory for functionals of class $C^{1}$, C. R. Acad. Sci. Paris Sér. I Math. 315 (1992) 883-888. MR 93i:58027

[9] B. Buffoni, A.R. Champneys and J.F. Toland, Bifurcation and coalescence of multi-modal homoclinic orbits for a Hamiltonian system, J. Dynamics and Diff. Eqns. 8 (1996), 221-281. MR 97m:58143

[10] C. Conley, Isolated invariant sets and the Morse index, C.B.M.S. Reg. Conf. Ser. Math. 38 (1978), published by the AMS. MR 80c:58009

[11] M.G. Crandall, P.H. Rabinowitz and L. Tartar, On a Dirichlet problem with a singular nonlinearity, Comm. Part. Diff. Eqns. 2 (1977), 193-222. MR 55:856

[12] R. Ghrist, J.B. Van den Berg and R.C.A.M. Vandervorst, Closed characteristics of fourthorder Twist systems via braids, Comptes Rendus Ac. Sc. Paris, 331 (2000), 861-865. MR 2001:06

[13] R. Ghrist, J.B. Van den Berg and R.C.A.M. Vandervorst, Morse theory on spaces of braids and Lagrangian dynamics, preprint (2001).

[14] J. Hulshof, J.B. Van den Berg and R.C.A.M. Vandervorst, Traveling waves for fourth-order semilinear parabolic equations, SIAM J. Math. Anal. 32 (2001), 1342-1374.

[15] W.D. Kalies and R.C.A.M. Vandervorst, Multitransition homoclinic and heteroclinic solutions of the extended Fisher-Kolmogorov equation, J. Diff. Eqns. 131 (1996), 209-228. MR 97h:34050

[16] W.D. Kalies and R.C.A.M. Vandervorst, Second order Lagrangians musings, in preparation.

[17] W.D. Kalies, J. Kwapisz and R.C.A.M. Vandervorst, Homotopy classes for stable connections between Hamiltonian saddle-focus equilibria, Comm. Math. Phys. 193 (1998), 337-371. MR 99g:34102

[18] W.D. Kalies, J. Kwapisz, J.B. Van den Berg and R.C.A.M. Vandervorst, Homotopy classes for stable periodic and chaotic patterns in fourth-order Hamiltonian systems, Comm. Math. Phys. 214 (2000), 573-592. CMP 2001:05

[19] J. Kwapisz, Uniqueness of the stationary wave for the extended Fisher-Kolmogorov equation, J. Diff. Eq. 165 (2000), 235-253. CMP 2000:15

[20] J. Kwapisz, (personal communication).

[21] J.D. Logan, Invariant variational principles, Math. Science Eng. 138, Academic Press 1977. MR 58:18024

[22] J.N. Mather, Existence of quasi-periodic orbits for twist diffeomorphisms of the annulus, Topology 21 (1982), 457-467.

[23] J. Milnor, Morse Theory, Ann. Math. Studies 51, Princeton University Press, 1963. MR 29:634 
[24] V.J. Mizel, L.A. Peletier and W.C. Troy, Periodic phases in second order materials, Arch. Rat. Mech. Anal. 145 (1998) 343-382. MR 99j:73011

[25] P. LeCalvez Propriété dynamique des diffeomorphismes de l'anneau et du tore, Astérique 204, 1991. MR 94d:58092

[26] L.A. Peletier and A.I. Rotariu-Bruma, Solitary wave solutions to a four-parameter model for water waves, preprint (1999).

[27] L.A. Peletier, A.I. Rotariu-Bruma and W.C. Troy, Pulse-like spatial patterns described by higher-order model equations, J. Diff. Eqns. 150 (1998), 124-187. MR 99j:35098

[28] L.A. Peletier and W.C. Troy, Spatial patterns described by the extended Fisher-Kolmogorov equation: kinks, Diff. Int. Eqns. 8 (1995), 1279-1304. MR 96c:35182

[29] L.A. Peletier and W.C. Troy, Spatial patterns described by the extended Fisher-Kolmogorov equation: periodic solutions, SIAM J. Math. Anal. 28(6) (1997), 1317-1353. MR 98k:35198

[30] L.A. Peletier and W.C. Troy, Multibump periodic traveling waves in suspension bridges, Proc. Roy. Soc. Edin. 128A (1998), 631-659. MR 99h:34038

[31] L.A. Peletier, W.C. Troy and J.B. Van den Berg Global branches of multi bump periodic solutions of the Swift-Hohenberg equation, Arch. Rat. Mech. Anal. 158 (2001), 91-153. CMP 2001:14

[32] J. Swift and P.C. Hohenberg, Hydrodynamic fluctuations at the convective instability, Phys. Rev. A 15 (1977), 319-328.

[33] J.B. Van den Berg, The phase-plane picture for a class of fourth-order conservative differential equations, J. Diff. Eq. 161 (2000), 110-153. MR 2000k:34074

Division of Theoretical Mechanics, University of Nottingham, Nottingham NG7 2RD, United Kingdom

E-mail address: Jan.Bouwe@nottingham.ac.uk

$U R L:$ http://spencer.thmech.nottingham.ac.uk/ pmzjb/

Mathematical Institute, Leiden University, P.O. Box 9512, 2300 RA Leiden, The NETHERLANDS

Current address: Center for Dynamical Systems and Nonlinear Studies, Georgia Institute of Technology, Atlanta, Georgia 30332-0190

E-mail address: rvander@math.gatech.edu

$U R L:$ http://www.math.gatech.edu/ ${ }^{\sim}$ rvander 